\title{
Unraveling the Mechanisms Underlying Irregularities in Inspiratory Rhythm Generation in a Mouse Model of Parkinson's Disease
}

\author{
${ }^{(}$Luiz M. Oliveira, ${ }^{1,2}$ Nathan A. Baertsch, ${ }^{2,5}$ Thiago S. Moreira, ${ }^{3}$ Jan-Marino Ramirez, ${ }^{2,4,5}$ and Ana C. Takakura ${ }^{1}$ \\ ${ }^{1}$ Department of Pharmacology, Instituto de Ciencias Biomedicas, Universidade de São Paulo, São Paulo 05508, Brazil, ${ }^{2}$ Center for Integrative Brain \\ Research, Seattle Children's Research Institute, Seattle, Washington 98101, ${ }^{3}$ Department of Physiology and Biophysics, Instituto de Ciencias \\ Biomedicas, Universidade de São Paulo, São Paulo 05508, Brazil, ${ }^{4}$ Department of Neurological Surgery, University of Washington, Seattle, \\ Washington 98101, and ${ }^{5}$ Department of Pediatrics, University of Washington, Seattle, Washington 98101
}

Parkinson's disease (PD) is a neurodegenerative disorder anatomically characterized by a progressive loss of dopaminergic neurons in the substantia nigra compacta $(\mathrm{SNpc})$. Much less known, yet clinically very important, are the detrimental effects on breathing associated with this disease. Consistent with the human pathophysiology, the 6-hydroxydopamine hydrochloride (6-OHDA) rodent model of PD shows reduced respiratory frequency $\left(f_{R}\right)$ and NK1r-immunoreactivity in the pre-Bötzinger complex (preBötC) and $\mathrm{PHOX}^{+} \mathrm{B}^{+}$neurons in the retrotrapezoid nucleus (RTN). To unravel mechanisms that underlie bradypnea in PD, we employed a transgenic approach to label or stimulate specific neuron populations in various respiratoryrelated brainstem regions. PD mice were characterized by a pronounced decreased number of putatively rhythmically active excitatory neurons in the preBötC and adjacent ventral respiratory column (VRC). Specifically, the number of Dbxl and Vglut $_{2}$ neurons was reduced by $47.6 \%$ and $17.3 \%$, respectively. By contrast, inhibitory Vgat $^{+}$neurons in the VRC, as well as neurons in other respiratory-related brainstem regions, showed relatively minimal or no signs of neuronal loss. Consistent with these anatomic observations, optogenetic experiments identified deficits in respiratory function that were specific to manipulations of excitatory (Dbx1/Vglut ${ }_{2}$ ) neurons in the preBötC. We conclude that the decreased number of this critical population of respiratory neurons is an important contributor to the development of irregularities in inspiratory rhythm generation in this mouse model of PD.

Key words: breathing; medulla; Parkinson's disease; photo-activation; pre-Bötzinger complex; retrotrapezoid nucleus

Significance Statement

We found a decreased number of a specific population of medullary neurons which contributes to breathing abnormalities in a mouse model of Parkinson's disease (PD).

Received Aug. 11, 2020; revised Mar. 3, 2021; accepted Mar. 9, 2021.

Author contributions: L.M.O., T.S.M., J.-M.R., and A.C.T. designed research; L.M.O. and N.A.B. performed research; L.M.O., N.A.B., T.S.M., J.-M.R., and A.C.T. analyzed data; L.M.O., N.A.B., T.S.M., J.-M.R., and A.C.T. wrote the paper.

This work was supported by the Serrapilheira Institute Grant Serra-1812-26431, São Paulo Research Foundation (FAPESP) Grants 2019/01236-4 (to A.C.T.) and 2015/23376-1 (to T.S.M.), the Conselho Nacional de Desenvolvimento Científico e Tecnológico (CNPq) Grant 408647/2018-3 (to A.C.T.), National Institutes of Health Grants K99 HL145004 and F32 HL134207 (to N.A.B.) and R01 HL126523 and R01 HL144801 (to J.-M. R.), FAPESP Fellowships 2015/18842-3, 2017/12266-6, and 2019/09323-3 (to L.M.O.), and CNPq Fellowships 302334/2019-0 (to T.S.M.) and 302288/2019-8 (to A.C.T.). We thank Dr. Christopher Del Negro for donation of $D b x 1^{c^{c e E R T 2}}$ mice, Dr. Eric Turner for sharing the stereotaxic apparatus, and Micah Greenberg for technician support.

The authors declare no competing financial interests.

Correspondence should be addressed to Ana C. Takakura at takakura@icb.usp.br.

https://doi.org/10.1523/JNEUROSCI.2114-20.2021

Copyright $\odot 2021$ the authors

\section{Introduction}

Neurodegenerative diseases, including Parkinson's disease (PD), are often associated with motor control dysfunctions including respiratory impairment (Seccombe et al., 2011; Baille et al., 2016). Yet, in contrast to resting tremor, stiffness and movement rigidity, bradykinesia and postural instability (Angel et al., 1970; Moreno Catalá et al., 2016), breathing abnormalities remain poorly understood and understudied despite their prominence in PD (Maria et al., 2003; Baille et al., 2016; Zhang et al., 2016). Clinical reports describe a variety of respiratory symptoms ranging from dyspnea, deficiencies in respiratory muscle contractility and coordination of thoracic musculature, rhythmicity dysfunction over the course of the disease, as well as daytime somnolence because of nocturnal hypoxia (Stocchi et al., 1998; Seccombe et al., 2011; Baille et al., 2016; Owolabi et al., 2016). These respiratory disturbances have significant consequences for quality of life. Perhaps most consequential are respiratory dysfunctions 
that lead to aspiration pneumonia, a major cause of death in PD (Simons, 2017; Tomita et al., 2018; Schiffer and Kendall, 2019). Thus, developing treatments that mitigate these respiratory abnormalities will be important for managing PD patients.

Breathing is a highly integrated behavior that involves multiple interacting brain regions. In mammals, including humans, inspiration is the dominant active phase of breathing. Inspiratory activity emerges from a region in the ventral medulla called the pre-Bötzinger complex (preBötC; Smith et al., 1991; Kam et al., 2013; Wang et al., 2014). This dynamic network is heterogeneous, and contains interacting subsets of inhibitory and excitatory neurons (Smith et al., 1998; Cregg et al., 2017; Hayes et al., 2017; Baertsch et al., 2018). Of particular importance for inspiratory rhythmogenesis are glutamatergic neurons derived from Dbx1expressing progenitors (i.e., Dbx1 neurons; Wang et al., 2014; Vann et al., 2018). Inspiration is followed by postinspiration. During this respiratory phase, muscles actively slow the passive release of air from the lungs (Feldman et al., 2013). The postinspiratory complex (PiCo), rostral to the preBötC, has been implicated in the generation of the postinspiratory rhythm (Anderson et al., 2016; Toor et al., 2019), with significant modulatory influences from the pons, including the Kölliker-Fuse (KF) nucleus (Stanić et al., 2018). Under conditions of high ventilatory demand, air is forcefully expelled from the lungs during a phase called "active expiration." The retrotrapezoid nucleus/parafacial respiratory group ( $\mathrm{RTN} / \mathrm{pFRG}$ ) in the ventral medulla is thought to contain rhythmically active neurons that drive active expiration (Marina et al., 2010; Huckstepp et al., 2015) as well as chemosensitive neurons that contribute to breathing responses to $\mathrm{CO}_{2}$ (Mulkey et al., 2004; Stornetta et al., 2006; Takakura et al., 2006; Abbott et al., 2009). Together, these interacting networks give rise to a highly dynamic and adaptable rhythmic breathing behavior. Thus, respiratory abnormalities associated with $\mathrm{PD}$ may involve multiple distinct region(s) within the brainstem.

In order to unravel the contributions of specific brain regions and mechanisms underlying respiratory dysfunction in PD, studies using animal models are necessary. Indeed, animal models support clinical observations. For example, depleting catecholaminergic neurons in the substantia nigra compacta $(\mathrm{SNpc})$ in a murine model of PD leads to bradypnea at rest and impairment of the ventilatory response to hypercapnia (Tuppy et al., 2015). The cellular-level and network-level mechanisms underpinnings of these breathing disturbances are not well understood and are likely complex. Initial studies in murine models of PD have noted reduced expression of the neurokinin-1 receptor (NK1-r), a marker for preBötC neurons, in the ventral medulla (Tuppy et al., 2015; Oliveira et al., 2017; FernandesJunior et al., 2018). Reduced expression has also been described for the RTN marker PHOX2B (Tuppy et al., 2015; Fernandes-Junior et al., 2018; Oliveira et al., 2019). Thus, there is some evidence for anatomic changes in brainstem respiratory control regions in murine models of $\mathrm{PD}$. The introduction of cell-type-specific transgenic approaches allows us to unravel which functionally defined respiratory neurons may degenerate in $\mathrm{PD}$, an important step toward understanding the cellular mechanisms underlying respiratory dysfunction in PD. Here, we used these approaches to characterize in greater detail the cell-type-specific anatomic and functional deficits within brainstem regions important for the generation and modulation of breathing.

\section{Materials and Methods}

Animals

Experiments were performed on 119 adult C57/Bl6 mice of either sex weighing between 25 and $30 \mathrm{~g}$. The animals had free access to water and food and were housed in a temperature-controlled $\left(22 \pm 1^{\circ} \mathrm{C}\right)$ facility with a $12 / 12 \mathrm{~h}$ light/dark cycle. All experimental and surgical procedures were conducted in accordance with the National Institutes of Health and to the defined guidelines by the Seattle Children's Research Institute (SCRI) animal care and use committee (protocol number 18819) and by the Institutional animal care and use committee at the University of São Paulo (protocol number 58/2017).

$D b \times 1^{\text {creERT2(+/-) }}$ mice were donated by Del Negro (College of William and Mary, VA); Vgat-ires-cre ${ }^{(+/+)}$(stock \#016962), Vglut $_{2}$-ires-cre ${ }^{(+/+)}$(stock \#028863), Chat-ires-cre ${ }^{(+/+)}$(stock $\# 006410)$, and PHOX2B-cre ${ }^{(+/-)}$(stock \#025436) breeders were obtained from The Jackson Laboratory. $D b \times 1^{\operatorname{CreERT2(+/+)}}$ and PHOX $2 B-c r e^{(+/+)}$breeder lines were generated at SCRI. To anatomic experiments, $\mathrm{cre}^{(+/+)}$mice were bred with homozygous cre-dependent enhanced green fluorescent protein (ZsGreen1)expressing mice (Ai6) inserted in the ROSA26 locus (stock \#007906) from The Jackson Laboratory. For optogenetic activation experiments, $c r e^{(+/+)}$mice were bred with homozygous mice containing a floxed STOP channelrhodopsin2 fused to a EYFP reporter (Ai32) donated by Hongkui Zeng (Allen Brain Institute, WA). Dbx $1^{\text {creERT2(+/+) }}$ dams were monitored plug checked and tamoxifen injected ( $24 \mathrm{mg} / \mathrm{kg}$, i.p., Sigma-Aldrich) on embryonic day (E)9.5. Tamoxifen, when intraperitoneally injected during gestation, binds to a mutated estrogen receptor (ER) fused to cre recombinase as a transgene (cre-ER), which only promotes cre-ER activation and expression in cells that present the cre-ER at the time of injection. Mice were typically born (Dbx1-cre; Ai32) after $20 \mathrm{~d}$ of gestation via C-section. All the lineages used in our study have been described in the literature (Anderson et al., 2016; Baertsch et al., 2018; Vann et al., 2018). Litters generated were separate and used randomly by the investigators.

\section{Recovery surgeries for PD mouse model}

Recovery surgery procedures are summarized in Figure 1. Injections of 6-hydroxydopamine hydrochloride (6-OHDA; Sigma) or vehicle ( $1 \mu \mathrm{g}$ ascorbic acid in $1 \mu \mathrm{l}$ of saline $0.9 \%$ ) were made while mice were submitted to general anesthesia with inhaled $2 \%$ isoflurane in $100 \%$ oxygen. Surgery was performed using standard aseptic technique. For chemical lesions, mice were fixed to a Robot Stereotaxic frame (Neurostar). Bilateral injections of 6 -OHDA $(10 \mu \mathrm{g} / \mu \mathrm{L} ; 0.5 \mu \mathrm{L})$ were made into the caudate putamen $(\mathrm{CPu})$ using the following coordinates: $0.4 \mathrm{~mm}$ caudal to bregma; $1.9 \mathrm{~mm}$ lateral to the midline and $3 \mathrm{~mm}$ below the skull surface. The coordinates and the dose of 6-OHDA used in the present study were selected based on the literature and previous experiments (Zhang et al., 2015; Goes et al., 2018; Oliveira et al., 2019). All injections were performed through a single-barrel needle with an external tip coupled to a Hamilton's syringe.

Following vehicle or 6-OHDA $\mathrm{CPu}$ injections, mice were implanted unilaterally with one $220 \mu \mathrm{m}$ or bilaterally with two $125-\mu \mathrm{m}$ fiber optics targeting the preBötC or the RTN using a $15^{\circ}$ rostral to caudal sagittal angle. We used the following coordinates for preBötC: $6.7 \mathrm{~mm}$ caudal to bregma, $1.2 \mathrm{~mm}$ lateral to the midline, and $4.8 \mathrm{~mm}$ below the skull surface; and for RTN: $6.3 \mathrm{~mm}$ caudal to bregma, $1.6 \mathrm{~mm}$ lateral to the midline, and 5.2 $\mathrm{mm}$ below the skull surface (Paxinos and Franklin, 2012). After 

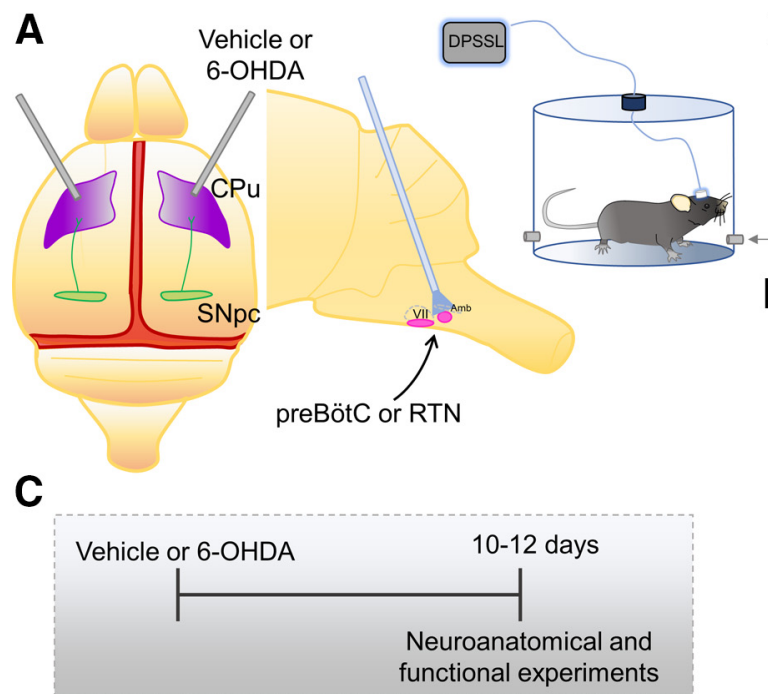

B Photostimulation:
1) Inspiration

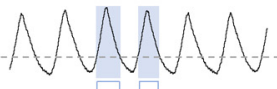
w

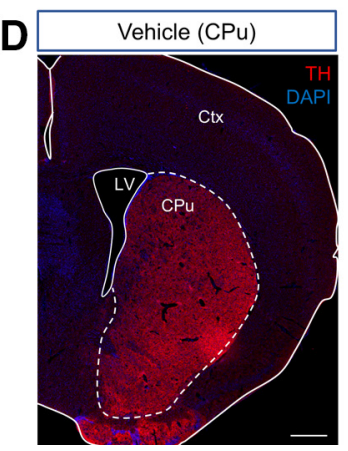

2) Expiration
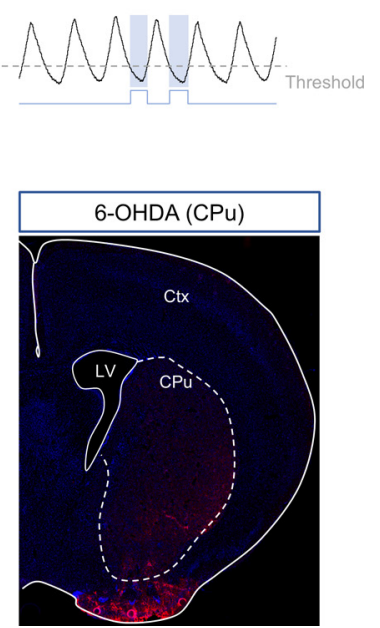

Figure 1. Design protocol. $\boldsymbol{A}$, Schematic view of vehicle or 6-OHDA injections into the (Pu, including the drug target (SNpc), the fiber optic location in the brainstem and the plethysmograph approach used in our experiments. Protocol $(\boldsymbol{B})$ and time course $(\boldsymbol{C})$ used in our study. $\boldsymbol{D}$, TH staining in the $\mathrm{CPu}$ in vehicle and a massive reduction of TH expression in the $\mathrm{CPu}$ of PDinduced animals. CPu, caudate putamen; Ctx, cortex; LV, lateral ventricle; preBötc, pre-Bötzinger complex; RTN, retrotrapezoid nucleus; SNpc, substantia nigra pars compacta. Scale bar: $600 \mu \mathrm{m}(\boldsymbol{D})$.

all surgical procedures, mice were treated with carpofren $(5 \mathrm{mg} /$ $\mathrm{kg} / \mathrm{d}$ for $2 \mathrm{~d}$ ) and maintained $10 \mathrm{~d}$ before anatomic or $10-12 \mathrm{~d}$ before functional experiments were performed. The toxin did not produce any readily observable behavioral effects.

\section{Gas consumption experiments}

Oxygen consumption $\left(\mathrm{V}^{2} \mathrm{O}_{2}\right)$ and $\mathrm{CO}_{2}$ release/production $\left(\mathrm{V}^{2} \mathrm{CO}_{2}\right)$ were assessed in unanesthetized adult mice in plethysmograph chambers $(300 \mathrm{ml})$ ventilated continuously with room air after acclimation phase (at least $30 \mathrm{~min}$ ). Gas was pulled through a small and horizontal column of drierite before being analyzed for the fractional concentrations of $\mathrm{O}_{2}$ and $\mathrm{CO}_{2}$ (model ML206, Gas analyzer, ADInstruments). The sensors were calibrated using known gas mixtures $\left(100 \% \mathrm{O}_{2}, 8 \% \mathrm{O}_{2}\right.$, and $7 \%$ $\mathrm{CO}_{2}$ ). A pump inside the gas analyzer pulled the air room at inflow rate of $200 \mathrm{ml} / \mathrm{min}$. The outputs were acquired for $30 \mathrm{~min}$ at $200 \mathrm{~Hz}$ by PowerLab system and Labchart software (version 8). The $\mathrm{V}^{2} \mathrm{O}_{2}$ has been calculated by Depocas and Hart formula (1957) for an open flow respirometry system with a downstream flowmeter: $\mathrm{V}^{2} \mathrm{O}_{2}=\left[\mathrm{F}_{\mathrm{IN}} \cdot\left(\mathrm{F}_{\text {insp }} \mathrm{O}_{2}-\mathrm{F}_{\text {exp }} \mathrm{O}_{2}\right)\right] /[1-(1-\mathrm{RQ})$. $\mathrm{F}_{\text {insp }} \mathrm{O}_{2}$ ], where $\mathrm{F}_{\mathrm{IN}}$ is the inflow rate of air in the chamber $(200$ $\mathrm{ml} / \mathrm{min}$ ), $\mathrm{F}_{\text {insp }} \mathrm{O}_{2}$ is the inspired $\mathrm{O}_{2}$ fraction (20.65\%, room air), RQ is assumed to be 0.85 and $\mathrm{F}_{\text {exp }} \mathrm{O}_{2}$ is the $\mathrm{O}_{2}$ expired fraction (acquired by the equipment; Depocas and Hart, 1957; Patrone et al., 2018). The $\mathrm{V}^{2} \mathrm{CO}_{2}$ was determined using a rearranged Depocas and Hart formula: $\mathrm{V}^{2} \mathrm{O}_{2}=\left[\mathrm{F}_{\mathrm{IN}} \cdot\left(\mathrm{F}_{\text {insp }} \mathrm{O}_{2}-\mathrm{F}_{\text {exp }} \mathrm{O}_{2}\right)-\right.$ $\left.\left(\mathrm{V}^{2} \mathrm{CO}_{2} \cdot \mathrm{F}_{\text {insp }} \mathrm{O}_{2}\right)\right] /\left(1-\mathrm{F}_{\text {insp }} \mathrm{O}_{2}\right)$ (Depocas and Hart, 1957; Withers, 1977).

\section{Unanesthetized plethysmograph experiments}

Respiratory parameters were recorded in unanesthetized adult mice using whole-body plethysmograph (Buxco) in 300-ml chambers ventilated continuously with normal air (79\% nitrogen and $21 \%$ oxygen) at a rate of $180-200 \mathrm{ml} / \mathrm{min}$ (Fig. 1). Volume calibration was performed during each experiment by injecting air $(1 \mathrm{ml})$ into the chamber. All experiments were performed at room temperature $\left(21-23^{\circ} \mathrm{C}\right)$. Mice were anesthetized with isoflurane briefly $(<30 \mathrm{~s})$, tethered to the fiber optic cable connected to a blue ( $447 \mathrm{~nm}$ ) laser and DPSS driver, and allowed at least $2 \mathrm{~h}$ to acclimate to the chamber environment.

To perform phase-dependent optogenetic stimulations, plethysmography waveforms were thresholded to trigger a transistortransistor logic (TTL) pulse during the rising or falling phase of each respiratory cycle (Clampex software). Inspiratory stimulations were triggered during the rising phase, whereas stimulations during the expiration were triggered during the falling phase (Fig. 1B; Baertsch et al., 2018; Vann et al., 2018). All the optogenetic experiments were performed while the mice were not sniffing, exploring or moving in the chamber. A limitation of this plethysmography approach is that it can be difficult to definitively discern respiratory phase transitions (e.g., onset and termination of inspiration). Therefore, several trials were performed during each experiment with the threshold positioned at different levels. As outlined in Figure 9, we used only those recordings for further analysis where the threshold was set to the very onset of either inspiration (Fig. 9A,C) or expiration (Fig. $9 B, D)$. All other recordings where the threshold was too high, and the stimulations occurred late in inspiration (Fig. 9E,C,G) or expiration (Fig. 9F,H), were excluded from our analysis.

\section{Anesthetized experiments}

The same mice used for unanesthetized plethysmography experiments were used in our anesthetized preparation. Surgical procedures were performed as previously described (Anderson et al., 2016; Baertsch et al., 2018). Briefly, adult mice were anesthetized with urethane $(1.5 \mathrm{mg} / \mathrm{kg}$, i.p.) and placed on a custom surgical table. The trachea was cannulated with a curved 24-G plastic tube. Mice were then allowed to spontaneously breathe $100 \% \mathrm{O}_{2}$ during the entire surgery and recordings. Adequate depth of anesthesia was determined via breathing frequency responses to toe pinch and adjusted if necessary, with supplemental urethane (intraperitoneal). The rostral trachea, esophagus and muscles were removed, to access the basal surface of the occipital bone. A small portion of the occipital bone and dura were removed to access the ventral medullary surface. Then, the brainstem surface was perfused with warmed $\left(\sim 36^{\circ} \mathrm{C}\right)$ aCSF $(118 \mathrm{~mm} \mathrm{NaCl}, 3.0 \mathrm{~mm}$ $\mathrm{KCl}, 325 \mathrm{~mm} \mathrm{NaHCO}, 1.0 \mathrm{~mm} \mathrm{NaH} \mathrm{PO}_{4}, 1.0 \mathrm{~mm} \mathrm{MgCl}_{2}, 1.5 \mathrm{~mm}$ 
$\mathrm{CaCl}_{2}$, and $30 \mathrm{~mm} \mathrm{D}$-glucose) equilibrated with carbogen (95\% $\mathrm{O}_{2}, 5 \% \mathrm{CO}_{2}$ ). Respiratory activity was recorded from the XII nerve using a fire-polished pulled glass pipette filled with aCSF attached to a suction electrode. The signal was amplified $(10,000 \times)$, filtered (low pass $300 \mathrm{~Hz}$, high pass $5 \mathrm{kHz}$ ), rectified, integrated, and digitized (Digidata $1550 \mathrm{~A}$, Molecular Devices). A glass fiber optic $(200 \mu \mathrm{m}$ in diameter $)$ connected to a blue $(447 \mathrm{~nm})$ laser and DPSS driver was placed in light contact with the brainstem and positioned for maximal effect on breathing frequency during light stimulation. Respiratory phase-specific stimulations were performed by thresholding integrated XII nerve activity as described above for unanesthetized plethysmography experiments (Fig. $1 B$ ).

\section{Optogenetic stimulations}

Subtle non-specific effects have been observed in $\mathrm{cre}^{-}$control mice when laser powers are $>8 \mathrm{~mW}$ (Baertsch et al., 2018). Thus, our optogenetic stimulations were performed using 7- to 8 -mW laser power. As controls for our optogenetic photostimulation experiments, we crossed $c r e^{(-1-)}$ damns with homozygous mice containing a floxed STOP ChR2 fused to a EYFP reporter (Ai32). This breeding scheme generates control mice that do not express ChR2. We subjected these mice to the same optogenetic stimulations as described above and did not observe changes in any measured breathing parameters in PD or control mice (Fig. 10).

\section{Histology}

At the end of experiments, all animals were deeply anesthetized with $4 \%$ isoflurane in $100 \%$ oxygen and perfused through the ascending aorta with $20 \mathrm{ml}$ of phosphate buffered saline (PB; $\mathrm{pH}$ 7.4) followed by $4 \%$ phosphate-buffered $(0.1 \mathrm{M}, \mathrm{pH} 7.4,20 \mathrm{ml})$ paraformaldehyde (Electron Microscopy Sciences). The brains were removed and stored in the perfusion fixative for $4 \mathrm{~h}$ at $4^{\circ} \mathrm{C}$ and sucrose $20 \%$ for $8 \mathrm{~h}$. Series of coronal sections $(25 \mu \mathrm{m})$ from the brains were cut using a cryostat and stored in cryoprotectant solution at $-20^{\circ} \mathrm{C}(20 \%$ glycerol plus $30 \%$ ethylene glycol in 50 $\mathrm{ml}$ phosphate buffer, $\mathrm{pH}$ 7.4) before histologic processing (Oliveira et al., 2017). All histochemical procedures were done using free-floating sections.

$\mathrm{TH}$ was detected using a polyclonal rabbit anti-TH antibody (AB152; Millipore; 1:1000) and GFAP was detected using a polyclonal mouse anti-GFAP (MAB360; Millipore; 1:2000) diluted in PB containing 2\% normal donkey serum (017-000-121, Jackson ImmunoResearch) and 0.3\% Triton X-100 and incubated for 24 h. Sections were subsequently rinsed in $\mathrm{PB}$ and incubated for $2 \mathrm{~h}$ in an Alexa Fluor 488 donkey anti-rabbit antibody (711-545-152; 1:250; Jackson ImmunoResearch) or Alexa Fluor 647 donkey anti-mouse (A31571; Life technologies; 1:400). For all secondary antibodies used, control experiments confirmed that no labeling was observed when primary antibodies were omitted. The sections were stained in a DAPI solution for $1 \mathrm{~min}$ and PB washed. The sections were mounted on slides in rostrocaudal sequential order, dried, and covered with fluoromount (00-4958-02; Thermo Fisher Scientific). Coverslips were affixed with nail polish.

Sections were also examined to confirm that fiber optics were placed in the intended location. When fiber optics were not correctly positioned, these mice were excluded from the analysis. As shown in Figure 11, according to the Paxinos and Franklin (2012) mouse atlas, when targeting the preBötC, unilateral or bilateral fiber tips were located just dorsal to the Nucleus ambiguus near bregma level $-6.84 \mathrm{~mm}, \sim 1300 \mu \mathrm{m}$ from the midline, and $\sim 700 \mu \mathrm{m}$ above the marginal layer (Fig. $11 A-F$ ). In the graphs, the distances used for the bilateral fiber optics implants were averaged. When the RTN was targeted, fiber tips were located near the facial nucleus at bregma level $-6.64 \mathrm{~mm}$, $\sim 1600 \mu \mathrm{m}$ from the midline, and $\sim 600 \mu \mathrm{m}$ above the marginal layer (Fig. $11 G-K$ ). There were no significant differences in fiber optic placement between any control and PD mouse groups.

\section{Cell counting, imaging, and data analysis}

A VS120-S6-W Virtual Slide Scanner (Olympus) was used to scan all the sections. Images were taken with a color camera (Nikon DS-Fi3). To restrict any influences on our counted results, the photomicrography and counting were performed by one blind researcher. ImageJ (version 1.41; National Institutes of Health) was used for cell counting and Canvas software (ACD Systems, v. 9.0) was used for line drawings. A one-in-two series of $25-\mu \mathrm{m}$ brain sections was used per mouse, which means that each section analyzed was $50 \mu \mathrm{m}$ apart. The area analyzed was delimited based on previously reports (Baertsch et al., 2018; Vann et al., 2018; mean of 116,228.1 $\mu^{2}$ ). The neurons were identified by a large cell body and different sizes and shapes were also considered. Dendrites, which are usually represented as numerous small projections extending from the cell body, were not counted. The sections were counted bilaterally, averaged and the numbers reported as mean \pm SEM. Section alignment related to the SNpc, RTN, VRC, nucleus of the solitary tract (NTS), $\mathrm{PiCo}$, and KF were relative to a reference section, as previously described (Lazarenko et al., 2009; Anderson et al., 2016; Vann et al., 2018; Oliveira et al., 2019) and based on Paxinos and Franklin (2012). To delimitate the ventral respiratory column (VRC) area, we used an ImageJ software to overlap a circle (radius of $60.82 \mu \mathrm{m}$ ) for all the pictures in the VRC. To delimitate this area, we used a dark field (non-fluorescent) to define anatomic and cytoarchitecture based on previous studies (Akins et al., 2017; Kottick et al., 2017; Baertsch et al., 2018; Vann et al., 2018; Oliveira et al., 2019). We choose to represent the VRC non-compartmentalized (i.e., BötC, preBötC, rVRG, and cVRG). First, the section alignment between brains was done relative to a reference section: the rostral segment of the VRC was identified by the last section with the caudal end of the facial motor neurons in each brain and brain levels were named according to the atlas of Paxinos and Franklin (2012). After that, to distinguish the VRC in each section, we used the nucleus ambiguous (Amb) and the ventral spinocerebellar tract as the main anatomic structures, both areas present a very clear anatomic limits and represents the dorsal and ventral limits of the VRC, respectively. The rostral-caudal portion also shows a very distinguish anatomic structure: Amb. Once the section that contains the rostral portion of Amb (more densely packed) is the section that contains the rostral portion of the VRC. In a caudal direction the Amb turns in a semi-compacta portion, and non-compacta even more caudal (Akins et al., 2017; Kottick et al., 2017; Baertsch et al., 2018; Vann et al., 2018) and the VRC was analyzed in all that extension. PiCo was anatomically characterized by immunohistological labeling, revealing choline acetyltransferase (ChAT)positive neurons located dorsomedial to Amb, located dorsal and caudal to the facial nucleus (Anderson et al., 2016; Toor et al., 2019). The neuroanatomical nomenclature employed during experimentation was defined by Paxinos and Franklin (2012).

\section{Statistics}

Statistical analysis was performed using Sigma Stat version 11.0 software (Jandel Corporation); $t$ test followed by Mann-Whitney 
sum-rank test or two-way ANOVA with Bonferroni's multiple comparison test were used when appropriate. Data are reported as mean \pm standard error of the mean. For all comparisons, $p<0.05$ was considered statistically significant.

\section{Results}

\section{PD mice with SNpc neurodegeneration have respiratory impairment}

To anatomically verify for the presence of a PD phenotype in our mouse model, the number of $\mathrm{TH}^{+}$cells in the $\mathrm{SNpc}$ were counted from all 6-OHDA $(n=59)$ and vehicle-injected $(n=60)$ mice used in this study (Fig. 2). TH-positive neurons were quantified in the SNpc between -2.75 and $-3.35 \mathrm{~mm}$ from bregma level (Fig. 2C). We observed a significant reduction of TH neurons in the $\mathrm{SNpc}$ in $\mathrm{PD}$-induced mice $(151.3 \pm 4.6$ vs control: $491.4 \pm 14.2 \mathrm{TH}^{+}$neurons; $t$ test, $t=1138.0, p<0.001$; Fig. $\left.2 D\right)$. There was also a substantial reduction in $\mathrm{TH}^{+}$varicosities expression in the $\mathrm{CPu}$ (Fig. $1 D$ ). To examine respiratory function, plethysmograph recordings from the same 6-OHDA injection PD mice and vehicle-injected control mice were analyzed. In $\mathrm{PD}$ injected mice, total ventilation $\left[\mathrm{VE}_{\mathrm{E}}=\mathrm{f}_{\mathrm{R}} \times\right.$ tidal volume $\left.\left(\mathrm{V}_{\mathrm{T}}\right)\right]$ was reduced $(0.54 \pm 0.03$ vs sham: $0.71 \pm 0.04 \mathrm{ml} / \mathrm{min} / \mathrm{g}$, $t$ test, $t=1172.0, p<0.001$; Fig. $2 E$ ) because of a slowing of breathing frequency $\left(f_{R} ; 154.6 \pm 6.0\right.$ vs sham: $205.9 \pm 3.7$ breaths $/ \mathrm{min}, t$ test, $t=2865.0, p<0.001$; Fig. $2 F)$ without a significant change in tidal volume $\left(\mathrm{V}_{\mathrm{T}} ; 3.60 \pm 0.17\right.$ vs sham: $3.43 \pm 0.16 \mu \mathrm{l} / \mathrm{g}, t$ test, $t=1987.0, p=0.628$; Fig. $2 G)$. Changes in breathing frequency were primarily because of a prolongation of expiratory time $\left(\mathrm{T}_{\mathrm{E}} ; 154.6 \pm 6.0 \mathrm{vs}\right.$ sham: $198.7 \pm 10.0 \mathrm{~ms}, t$ test, $t=1407.0, p<0.001$; Fig. $2 I$ ) without changes in inspiratory time $(177.0 \pm 7.4$ vs sham: $176.7 \pm 5.1 \mathrm{~ms}, t$ test, $t=2065.0, p=0.891$; Fig. $2 H$ ). Average frequency distributions of breathing frequency, tidal volume, and expiratory time are shown in Figure $2 J-L$, respectively. Breathing frequency also became more irregular in PD-induced mice $(0.050 \pm 0.007$ vs sham: $0.024 \pm 0.004, t$ test, $t=1211.0, p<0.001$; Fig. $2 N$ ) without significant changes in the regularity of $\mathrm{VE}_{\mathrm{E}}, \mathrm{V}_{\mathrm{T}}, \mathrm{T}_{\mathrm{I}}$, or $\mathrm{T}_{\mathrm{E}}$ (Fig. $2 \mathrm{M}, \mathrm{O}-\mathrm{Q}$, respectively).

Further, $\mathrm{V}^{2} \mathrm{O}_{2}$ and $\mathrm{V}^{2} \mathrm{CO}_{2}$ was evaluated in a separate experimental group ( $n=8$; Fig. $2 R-T$ ). Our results shows that there was a reduction of $\mathrm{V}^{2} \mathrm{O}_{2}(23.42 \pm 4.11$ vs sham: $60.96 \pm 4.30 \mathrm{ml} /$ min $\mathrm{kg}^{-1}$, two-way ANOVA, $F_{(1,15)}=14.828, p<0.001$; Fig. $1 S$ ) and $\mathrm{V}^{2} \mathrm{CO}_{2}$ after PD-induction $(21.42 \pm 3.17$ vs sham: $53.15 \pm 3.77 \mathrm{ml} / \mathrm{min} \mathrm{kg}^{-1}$, two-way ANOVA, $F_{(1,15)}=13.377$, $p<0.001$; Fig. 2T). Together, these experiments confirm that PD-induced mice have substantial respiratory impairments (Tuppy et al., 2015; Fernandes-Junior et al., 2018; Oliveira et al., 2019), primarily because of longer and more irregular periods between inspiratory efforts.

\section{The VRC of PD mice contains fewer glutamatergic Dbx1 neurons}

Excitatory synaptic interactions among recurrently connected glutamatergic neurons is a fundamental anatomic feature thought to be critical for inspiratory rhythmogenesis within the VRC (Carroll and Ramirez, 2013; Cui et al., 2016). To explore how this anatomic substrate may be disrupted in PD, we compared the number of $\mathrm{Vglut}_{2}{ }^{+}$neurons along the VRC between -6.56 and $-7.96 \mathrm{~mm}$ from bregma level in 6-OHDA-injected PD mice versus vehicle-injected controls. Strikingly, we found that the number of glutamatergic neurons was reduced along the VRC (2724 \pm 59 vs control: $3455 \pm 84$ Vglut $_{2}{ }^{+}$neurons; $t$ test, $t=7113, p<0.001$; Fig. $3 A, B)$. This reduction in glutamatergic neurons remained highly significant when presumably nonrhythmically active $\mathrm{TH}^{+} / \mathrm{Vglut}_{2}{ }^{+} \mathrm{C} 1$ neurons were excluded from the analysis (data not shown). However, there was also a smaller but significant reduction in $\mathrm{TH}^{+}$glutamatergic neurons (298 \pm 21 vs control: $382 \pm 36 \mathrm{TH}^{+}$neurons; $t$ test, $t=3.3$, $p=0.016$; data not shown) in the VRC of PD-induced mice compared with control animals.

Next, we assessed the anatomic integrity of Dbx1 neurons, a specific subpopulation of glutamatergic neurons critical for rhythmogenesis and frequency control within the preBötC (Cui et al., 2016; Akins et al., 2017; Baertsch et al., 2018). Consistent with our observations in Vglut $_{2}$-cre;Ai6 mice, we observed a significant reduction in the number of $\mathrm{Dbxl}^{+}$cells along the VRC of PD mice (1064 \pm 14 vs control: $2461 \pm 59 \mathrm{Dbxl}^{+}$cells; $t$ test, $t=10.0, p=0.029$; Fig. $3 C, D)$. Since the transcription factor Dbx1 is also expressed in some glia (Akins et al., 2017; SheikhBahaei et al., 2018), we performed immunostaining for GFAP to distinguish neurons from astrocytes. We found that the number of Dbx1 neurons $\left(\mathrm{GFAP}^{-}\right)$was significantly reduced within the VRC (1098 \pm 32 vs control: $1967 \pm 11 \mathrm{Dbxl}^{+}$neurons; $t$ test, $t=25.765, p<0.001$; Fig. 3G,H1) including the preBötC region. We also noted a smaller but significant reduction in the number of Dbx1-expressing glia $\left(\mathrm{GFAP}^{+}\right)$within the VRC of PD mice (196 \pm 12 vs control: $437 \pm 16$; $t$ test, $t=12.089, p<0.001$; Fig. $3 \mathrm{H} 2$ ), which was consistent with a significant reduction in the number of total GFAP ${ }^{+}$cells within the VRC $(254 \pm 14$ vs control: $647 \pm 68$; $t$ test, $t=5.70, p=0.005$; Fig. 3 H3).

Approximately $40-50 \%$ of the neurons that participate in the inspiratory rhythm are inhibitory (Janczewski et al., 2013; Baertsch et al., 2018), and these neurons play important roles in regulating breathing frequency and pattern (Janczewski et al., 2013; Sherman et al., 2015; Baertsch et al., 2018). Therefore, we next assessed anatomic deficits in the number of inhibitory VRC neurons using Vgat-cre;Ai6 mice with specific labeling of inhibitory glycinergic and/or GABAergic neurons (Baertsch et al., 2018). In contrast to excitatory Dbx1 or Vglut $_{2}$ neurons, we found no differences in the number $\mathrm{Vgat}^{+}$neurons along the VRC of PD mice when compared with controls $\left(3463 \pm 54\right.$ vs control: $3647 \pm 59$ Vgat $^{+}$cells; $t$ test, $t=2.305, p=0.061$; Fig. $3 E, F)$.

We also examined the anatomic integrity of glutamatergic neurons in the pontine KF nucleus, implicated in the modulation of breathing frequency (Dutschmann et al., 2021), and ChAT neurons within the PiCo (Anderson et al., 2016; Toor et al., 2019). Surprisingly, the number of $\mathrm{Vglut}_{2}{ }^{+}$cells in KF, was also reduced in $\mathrm{PD}$ animals ( $815 \pm 17$ vs control: $958 \pm 38 \mathrm{Vglut}_{2}{ }^{+}$ neurons; $t$ test, $t=3.368, p=0.01$; Fig. $4 B, D)$. Also, the total number of $\mathrm{ChAT}^{+}$cells in the PiCo was slightly reduced in PDinduced mice relative to vehicle-injected controls (185 \pm 9 vs control: $232 \pm 19 \mathrm{Chat}^{+}$neurons; $t$ test, $t=23.0, p=0.02$; Fig. $4 A, C)$. To determine whether the neuronal death process is restricted to the ventral medullary respiratory areas, we also counted Vglut $2^{+}$cells in the caudal and intermediate portion of the NTS (cNTS and iNTS, respectively). As represented in the Figure 4 , the total number of neurons in the cNTS ( $p=0.736, t=$ $-0.349)$ and iNTS $(p=0.793, t=-0.272)$ was not different comparing control and PD-induced animals. Collectively, these histologic data suggest that PD mice are characterized by specific neuroanatomical deficits in respiratory-related regions of the brainstem that are most prominent within the VRC, and primarily involve glutamatergic $\mathrm{Dbx1}{ }^{+}$neurons that are critical for generating the inspiratory rhythm (Cui et al., 2016; Vann et al., 2018). 

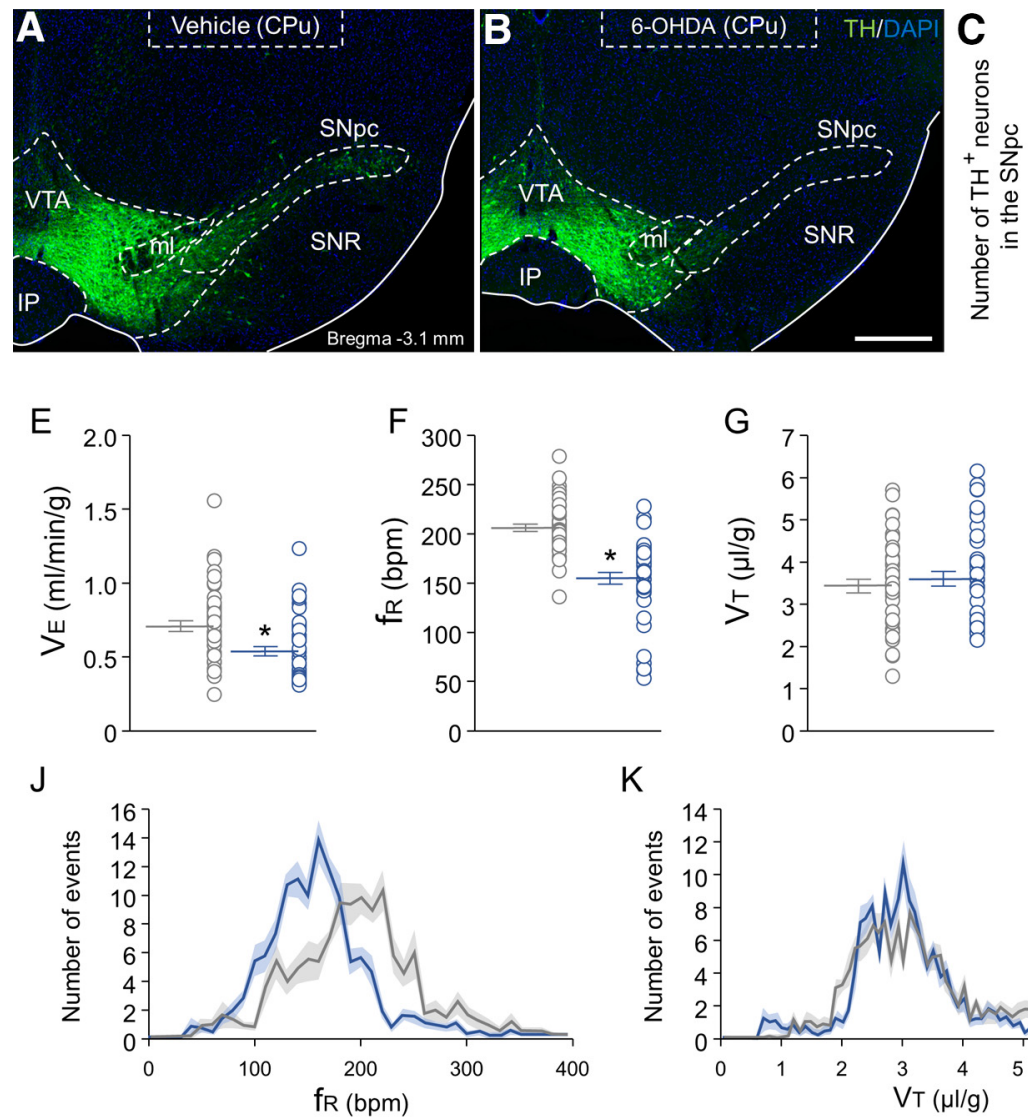

$\mathrm{K}$
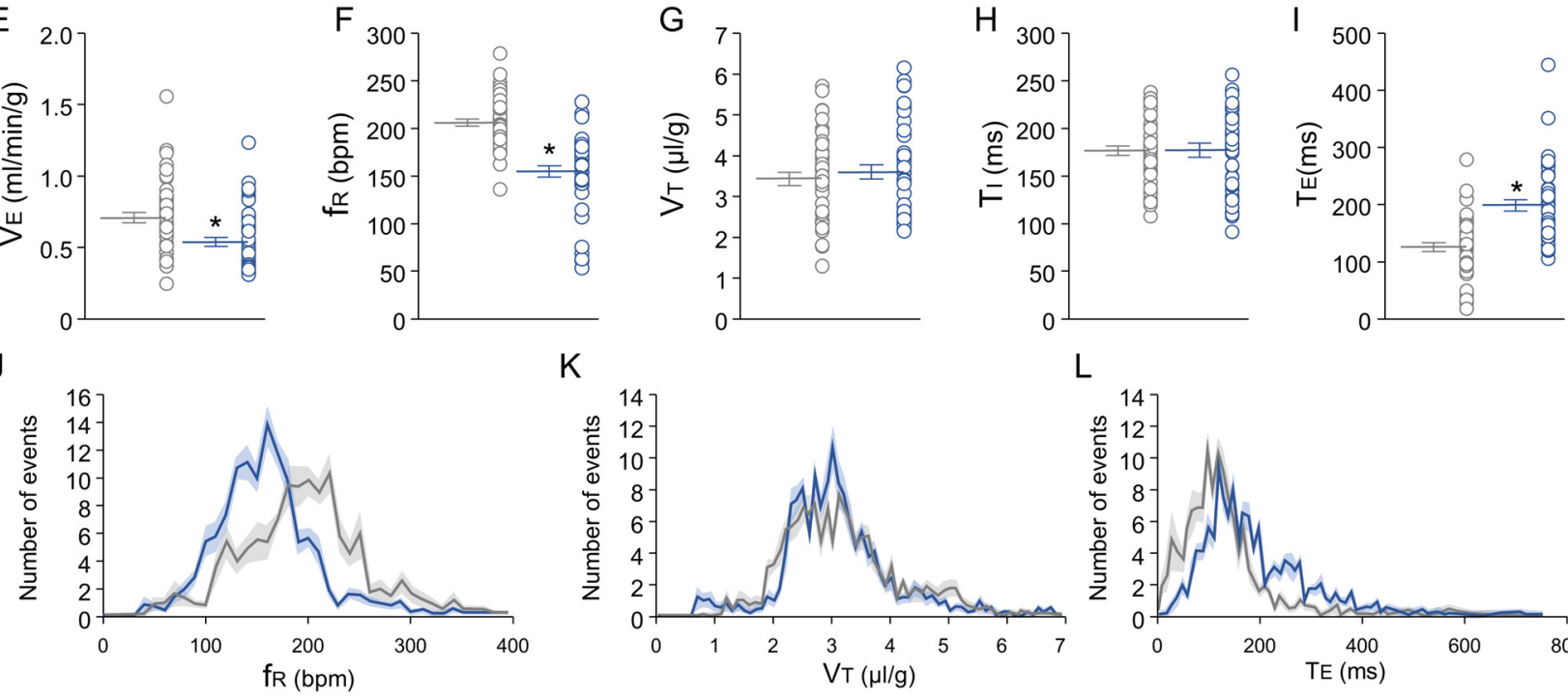

L

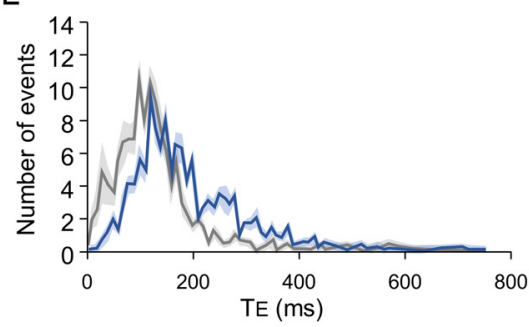

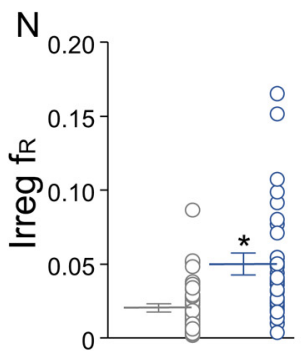
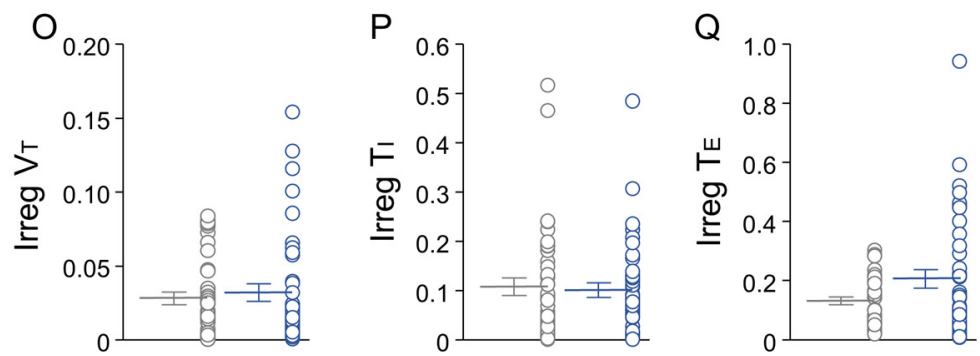

$\mathrm{R}$
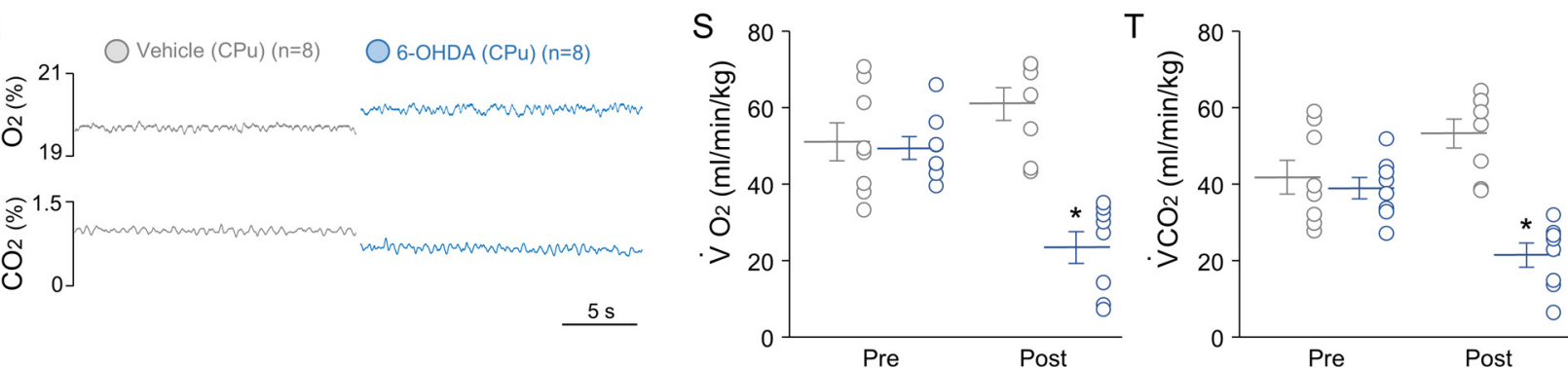

Figure 2. Anatomical and ventilatory disturbances in PD mice model. $\boldsymbol{A}, \boldsymbol{B}$, Photomicrographs showing a reduction in catecholaminergic neurons in the SNpc. Rostro-caudal distribution ( $\boldsymbol{C}$ and mean $(\boldsymbol{D})$ of $\mathrm{TH}^{+}$neurons after bilateral injection of 6-OHDA or vehicle into the $\left(\mathrm{Pu}\left(n=59-60 /\right.\right.$ group). Changes in $(\boldsymbol{E})$ ventilation $\left(\mathrm{VE} \mathrm{E}_{\mathrm{E}}\right),(\boldsymbol{F})$ respiratory frequency $\left(\mathrm{f}_{\mathrm{R}}\right),(\boldsymbol{G})$ tidal volume $\left(\mathrm{V}_{\mathrm{T}}\right),(\boldsymbol{H})$ inspiratory time $\left(\mathrm{T}_{\mathrm{I}}\right)$, and $(\boldsymbol{I})$ expiratory time $\left(\mathrm{T}_{\mathrm{E}}\right)$ in unanesthetized mice at room air $10 \mathrm{~d}$ after bilateral injection of 6-OHDA or vehicle into the $(\mathrm{Pu}$. Event distribution of $(\boldsymbol{J})$ respiratory frequency $\left(\mathrm{f}_{\mathrm{R}}\right),(\boldsymbol{K})$ tidal volume $\left(\mathrm{V}_{\mathrm{T}}\right)$, and $(\boldsymbol{L})$ expiratory time $\left(\mathrm{T}_{\mathrm{E}}\right)$ in unanesthetized mice at room air $10 \mathrm{~d}$ after bilateral injection of 6-OHDA or vehicle into the $(\mathrm{Pu}$. Irregularity score in $(M)$ ventilation $\left(\mathrm{VE}_{\mathrm{E}}\right),(\boldsymbol{N})$ respiratory frequency $\left(\mathrm{f}_{\mathrm{R}}\right),(\mathbf{O})$ tidal volume $\left(\mathrm{V}_{\mathrm{T}}\right),(\boldsymbol{P})$ inspiratory time $\left(\mathrm{T}_{\mathrm{B}}\right)$, and $\left.\mathbf{Q}\right)$ expiratory time $\left(\mathrm{T}_{\mathrm{E}}\right)$ in unanesthetized mice at room air $10 \mathrm{~d}$ after bilateral injection of 6 OHDA or vehicle into the $\mathrm{CPu}$; $t$ test followed by Mann-Whitney sum-rank test, $p<0.05$. $\boldsymbol{R}$, Representative recordings of $\mathrm{O}_{2}$ and $\mathrm{CO}_{2}$ expired fraction from two mice (vehicle-injected and PDinduced mice). Changes in $(S) \mathrm{V}^{2} \mathrm{O}_{2}$ and $(\boldsymbol{T}) \mathrm{V}^{2} \mathrm{CO}_{2}$ in unanesthetized mice at room pre and $10 \mathrm{~d}$ postbilateral injection of 6-OHDA or vehicle into the (Pu. ( $n=8 /$ group). Two-way ANOVA, followed by Newman-Keuls test, $p<0.05$. SNR, substantia nigra pars reticulata; SNpc, substantia nigra pars compacta; ml, medial lemniscus; VTA, ventral tegmental área; IP, interpeduncular nucleus. Scale bars: $500 \mu \mathrm{m}(\boldsymbol{A}, \boldsymbol{B})$. 

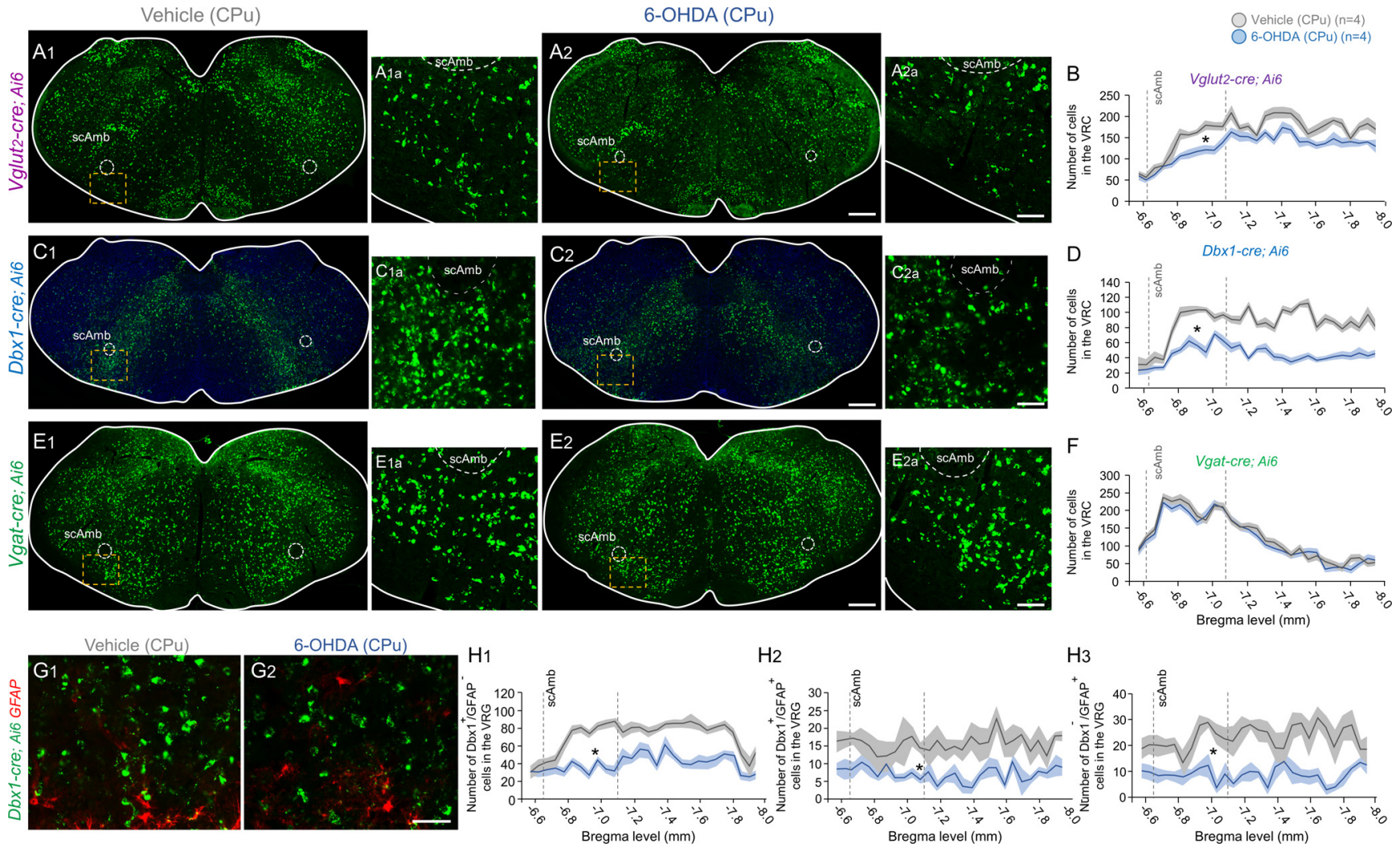

Figure 3. Changes of glutamatergic, Dbx1, and GABAergic neurons and GFAP cells in the VRC after bilateral injection of 6-OHDA or vehicle into the CPu. $A$, Photomicrographs showing a reduction of Vglut $_{2}^{+}$neurons in the medulla ventrolateral portion (bregma level: $-7.2 \mathrm{~mm}$ ). $\boldsymbol{B}$, Rostro-caudal distribution of $\mathrm{Vglut}_{2}{ }^{+}$neurons in VRC. $\boldsymbol{C}$, Photomicrographs showing a reduction of $\mathrm{Dbx} 1^{+}$neurons in the medulla ventrolateral portion. $\boldsymbol{D}$, Rostro-caudal distribution of $\mathrm{Dbx} 1^{+}$neurons in VRC. $E$, Photomicrographs showing no change of Vgat ${ }^{+}$neurons in the medulla ventrolateral portion. $\boldsymbol{F}$, Rostro-caudal distribution of $\mathrm{Vgat}^{+}$neurons in VRC; after bilateral injection of 6-OHDA (2) or vehicle (1) into the CPu ( $n=4 /$ group). G, Photomicrographs showing a reduction of Dbx $1^{+} / \mathrm{GFAP}^{+}$cells in the medulla ventrolateral portion. Rostro-caudal distribution of $\left.(\boldsymbol{H} 1) \mathrm{Dbx} 1^{+} / \mathrm{GFAP}^{-}, \mathrm{H} 2\right) \mathrm{Dbx}^{+} / \mathrm{GFAP}^{+}$and $(\mathrm{HZ}) \mathrm{Dbx}^{-} / \mathrm{GFAP}^{+}$cells after bilateral injection of 6-OHDA or vehicle into the (Pu ( $n=4 /$ group). Two-way ANOVA, followed by Newman-Keuls test, $p<0.05$. sc Amb, ambiguus nucleus semi-compact pars. Scale bars: $400 \mu \mathrm{m}$ (A2-E2; applies to A1, A2, C1, C2, E1, E2), $100 \mu \mathrm{m}$ (A2a; applies to A1a, A2a, C1a, C2a, E1a, E2a), and $50 \mu \mathrm{m}$ (G2; applies to G1, G2).

\section{Respiratory phase-dependent functional deficits of excitatory preBötC neurons in PD mice}

To further explore the functional consequences of the anatomic changes observed in PD mice, we used an optogenetic approach to selectively manipulate the activity of localized respiratoryrelated cell populations. In a first series of optogenetic experiments, we generated mice that specifically express $\mathrm{ChR} 2$ in glutamatergic Vglut $_{2}{ }^{+}$neurons. These Vglut $_{2}$-ChR2 mice were randomly separated and received injections of 6-OHDA or vehicle into the $\mathrm{CPu}$ ( $n=5$ /group) and an optical fiber was placed unilaterally to illuminate the preBötC (Fig. 1A); 10-12 d after surgery, photostimulation experiments were performed in unanesthetized mice while respiratory activity was monitored using plethysmography. In vehicle-injected control animals, light stimulations during the inspiratory phase significantly reduced by $20.2 \%$ the respiratory frequency $(165.2 \pm 8.5$ vs baseline: $206.9 \pm 5.3$ breaths $/ \mathrm{min}$, two-way ANOVA, $F_{(1,9)}=11.995$, $p=0.041$; Fig. $5 A, B)$. However, the same stimulation paradigm in PD mice caused a more modest reduction (10.1\%) in respiratory frequency that was not statistically significant $(130.6 \pm 8.1$ vs baseline: $145.2 \pm 4.2$ breaths/min, two-way ANOVA, $F_{(1,9)}=$ $11.995, p=0.164$; Fig. $5 A, B)$. The same animals were also subjected to photostimulations occurring during the expiratory phase, which increased respiratory frequency in both control (226.1 \pm 7.3 vs baseline: $202.2 \pm 1.4$ breaths $/ \mathrm{min}$, two-way ANOVA, $\left.F_{(1,9)}=19.348, p=0.002\right)$ and PD mice $(201.2 \pm 7.0$ vs baseline: $171.3 \pm 6.1$ breaths/min, two-way ANOVA, $F_{(1,9)}=$
19.348, $p=0.002$; Fig. $5 C, D$ ), corresponding to speeding up $\mathrm{f}_{\mathrm{R}}$ by $11.8 \%$ in vehicle-injected and $17.4 \%$ in $\mathrm{PD}$-induced mice.

Next, we performed similar photostimulation experiments in an anesthetized preparation to more accurately determine respiratory phase transitions by recording inspiratory activity directly from the XII nerve (Baertsch et al., 2018). Similar to our observations in unanesthetized animals, $\mathrm{f}_{\mathrm{R}}$ was slightly, but significantly, reduced by $16.0 \%$ during inspiratory photostimulation in control mice (79.6 \pm 4.4 vs baseline: $94.8 \pm 6.2$ breaths $/ \mathrm{min}$, two-way ANOVA, $\left.F_{(1,9)}=7.232, p=0.036\right)$; however, this effect was not significant (14.7\%) in PD mice $(60.0 \pm 2.5$ vs baseline: $70.3 \pm 7.5$ breaths $/ \mathrm{min}$, two-way ANOVA, $F_{(1,9)}=1.083$, $p=0.369$; Fig. $5 E, F)$. In both control and PD mice, expiratory photostimulations increased respiratory frequency to a similar rate (control: $123.1 \pm 1.4$ vs baseline: $93.9 \pm 8.5$ breaths $/ \mathrm{min}$ and PD: $110.6 \pm 7.2$ vs baseline: $66.4 \pm 7.2$ breaths $/ \mathrm{min}$, two-way ANOVA, $F_{(1,9)}=16.277, p=0.006$; Fig. $\left.5 G, H\right)$, which corresponded to a $66.6 \%$ increase in $\mathrm{f}_{\mathrm{R}}$ in $\mathrm{PD}$-induced mice compared with $31.1 \%$ in control mice.

In a second series of optogenetic experiments, we targeted Dbx1 neurons in the preBötC. Adult Dbx1-ChR2 mice were randomly chosen for injections of 6-OHDA or vehicle into the $\mathrm{CPu}$ ( $n=5$ /group) followed by unilateral implantation of a fiber optic at the level of the preBötC. As previously observed (Wang et al., 2014; Baertsch et al., 2018; Vann et al., 2018), respiratory phasedependent stimulations of Dbx1 neurons resulted in similar, but generally more modest effects, when compared with stimulations 

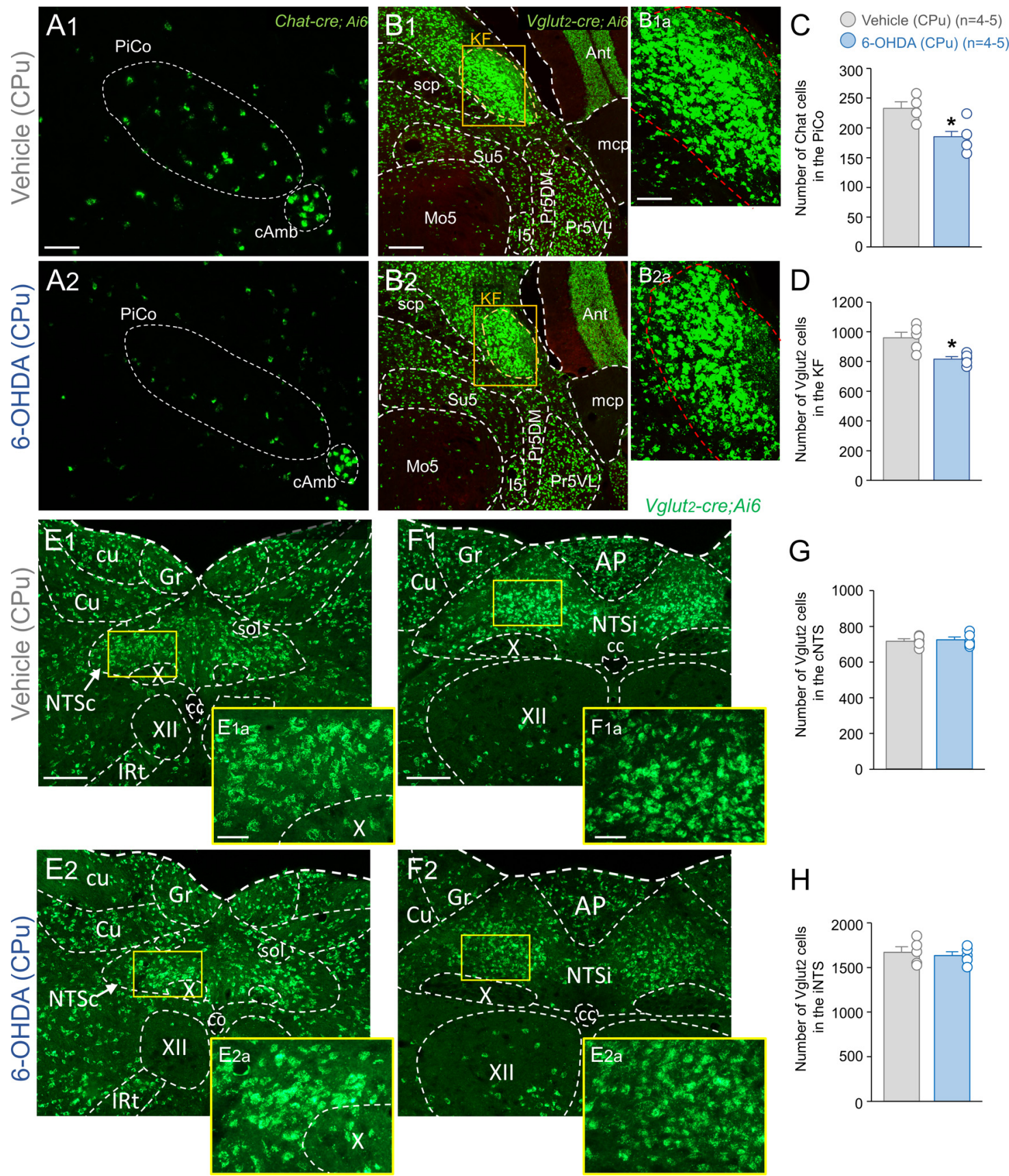

Figure 4. Changes of cholinergic neurons in PiCo and glutamatergic neurons in KF, CNTS, and iNTS after bilateral injection of 6-OHDA or vehicle into the CPu. A, Photomicrographs showing a reduction of Chat ${ }^{+}$neurons in the medulla ventromedial portion. $\boldsymbol{B}$, Photomicrographs showing a reduction of $\mathrm{Vglut}_{2}{ }^{+}$neurons in $\mathrm{KF}$ area. Number of $(\boldsymbol{C}) \mathrm{Chat}^{+}$neurons in PiCo and $(\boldsymbol{D})$ $\mathrm{Vglut}_{2}{ }^{+}$neurons in KF after bilateral injection of 6-OHDA or vehicle into the CPu $\left(n=4-5 /\right.$ group). Photomicrographs showing no changes in the number of Vglut ${ }^{+}{ }^{+}$neurons in the $(\boldsymbol{E})$ cNTS and $(\boldsymbol{F})$ iNTS after bilateral injection of 6-OHDA or vehicle into the $\mathrm{CPu}\left(n=5 / \mathrm{group}^{2}\right.$. Vglut ${ }_{2}{ }^{+}$neurons in $(\boldsymbol{G}) \mathrm{CNTS}$ and $(\boldsymbol{H})$ iNTS after bilateral injection of 6-OHDA or vehicle into the (Pu ( $n=4-5 /$ group); $t$ test followed by Mann-Whitney sum-rank test, $p<0.05$. CAmb, ambiguus nucleus compact pars; scp, superior cerebellar peduncle; Su5, supratrigeminal nucleus; M05, motor trigeminal nucleus; Ant, anterior lobe cerebellum; mcp, middle cerebellar peduncle; 15, intertrigeminal nucleus; Pr5DM, principal sensory trigeminal nucleus; Pr5VL, principal sensory trigeminal nucleus; $\mathrm{Cu}$, cuneate nucleus; cu, cuneate fasciculus; Gr, gracile nucleus; $\mathrm{X}$, dorsal motor nucleus of vagus; sol, solitary tract; $\mathrm{Cc}$, central canal; XII, hypoglossal nucleus; IRt, intermediate

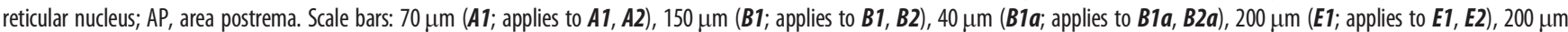
(F1; applies to F1, F2), $40 \mu \mathrm{m}$ (E1a; applies to E1a, E2a), and $40 \mu \mathrm{m}$ (F1a; applies to F1a, F2a).

of glutamatergic $\left(\mathrm{Vglut}_{2}{ }^{+}\right)$neurons in general (compare Figs. 5 and 6). Indeed, in unanesthetized mice, reductions in respiratory frequency during unilateral stimulation of Dbx1 neurons during the inspiratory phase were not statistically significant in either control [208.4 \pm 7.9 vs baseline: $216.9 \pm 3.8$ breaths/ $\min (3.9 \%)$, two-way ANOVA, $\left.F_{(1,9)}=0.966, p=0.364\right]$ or PD mice [170.4 \pm 8.4 vs baseline: $181.2 \pm 4.4$ breaths/min (5.9\%), twoway ANOVA, $F_{(1,9)}=0.966, p=0.364$; Fig. $\left.6 B\right]$. In addition, although trends were similar to our observations in $\mathrm{Vglut}_{2} ; \mathrm{ChR} 2$ mice, increases in frequency in response to stimulations of Dbx1 preBötC neurons during the expiration did not reach statistical significance (Fig. 6C,D).

However, when the same mice were anesthetized and XII nerve recordings were performed, differences in respiratory response to phase-dependent stimulations became more apparent between control and PD mice. Specifically, during 


\section{Unanesthetized}

A
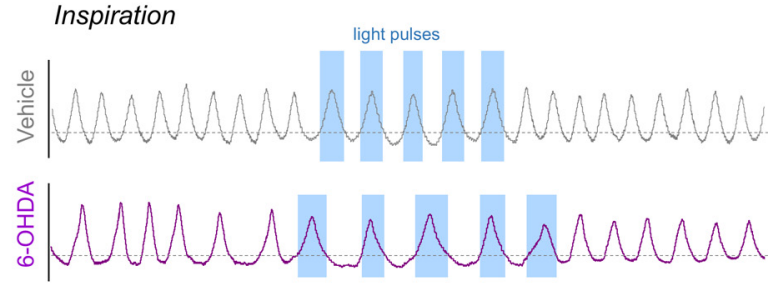

$\mathrm{B}_{1}$
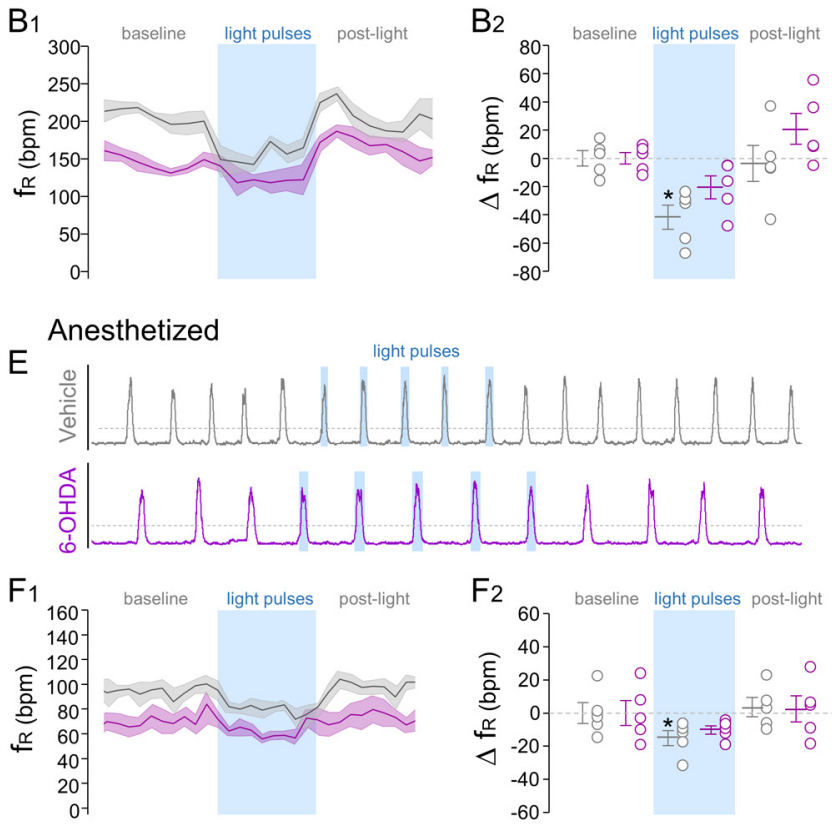

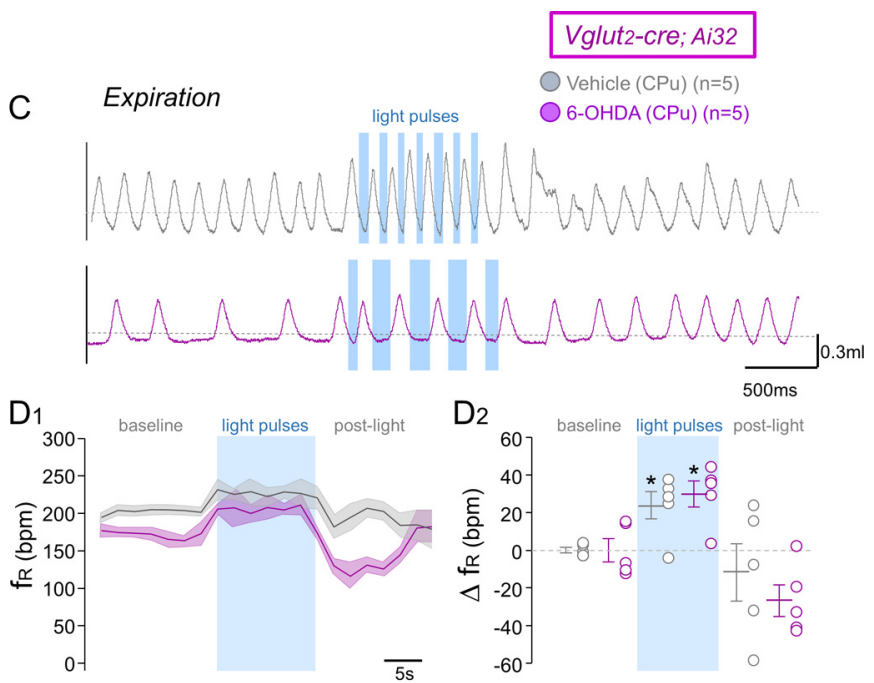

G
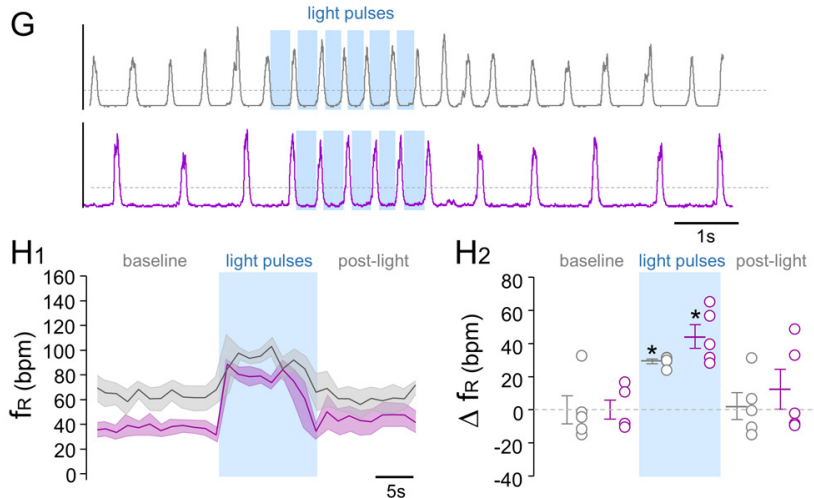

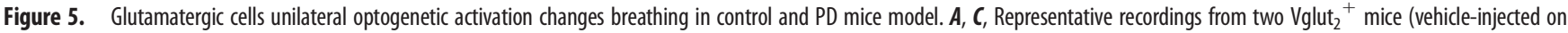
the top and PD-induced mice on the bottom) during optogenetic activation during $(\boldsymbol{A})$ inspiration and $(\boldsymbol{C})$ expiration. Changes in $(\boldsymbol{B}) \mathrm{f}_{\mathrm{R}}$ at inspiration and $(\boldsymbol{D})$ expiration photostimulation in unanesthetized mice at room air 10-12 d after bilateral injection of 6-0HDA or vehicle into the CPu ( $n=5 /$ group). B2, D2, 30-s recording average before, during, and 30-s average after photostimulation. $\boldsymbol{E}, \mathbf{G}$, Representative recordings from two anesthetized Vglut $_{2}{ }^{+}$mice (vehicle-injected on the top and PD-induced mice on the bottom) during optogenetic activation during $(\boldsymbol{E})$ inspiration and $(\boldsymbol{G})$ expiration. Changes in $\mathrm{f}_{\mathrm{R}}$ of $V$ glut $\mathrm{t}_{2}$-ChR2 animals during $(\boldsymbol{F})$ inspiration and $(\boldsymbol{H})$ expiration. $\boldsymbol{F 2}, \boldsymbol{H 2}$, 20-s recording average before, during, and 20-s average after photostimulation ( $n=5$ /group). Two-way ANOVA, followed by Newman-Keuls test, $p<0.05$.

inspiratory photostimulations, control $\mathrm{Dbx1-ChR2} \mathrm{mice} \mathrm{exhib-}$ ited a significant slowing of respiratory frequency by $15.4 \%$ $(82.9 \pm 2.8$ vs baseline: $98.0 \pm 1$. breaths/min, two-way ANOVA, $\left.F_{(1,9)}=51.97, p=0.003\right)$, whereas $\mathrm{PD}$ mice did not $(7.9 \%$; $65.2 \pm 2.5$ vs baseline: $70.8 \pm 0.5$ breaths/min, two-way ANOVA, $F_{(1,9)}=2.48, p=0.289$; Fig. $\left.6 E, F\right)$. And, when photostimulations occurred during the expiration, breathing frequency was significantly increased by $17.2 \%$ in PD mice $(85.7 \pm 1.7$ vs baseline: $73.1 \pm 1.0$ breaths $/ \mathrm{min}$, two-way ANOVA, $F_{(1,9)}=20.835$, $p<0.001$; Fig. $6 E, F)$, but was not $(13.3 \%)$ in controls $(107.0 \pm 4.0$ vs baseline: $94.4 \pm 7.5$ breaths $/ \mathrm{min}$, two-way ANOVA, $F_{(1,9)}=2.394, p=0.133$; Fig. $\left.6 G, H\right)$. Collectively, our data suggest that $\mathrm{PD}$ reduces specifically the number of excitatory preBötC neurons and that stimulation of these excitatory neurons elicit in PD a greater increase in the rate of breathing when activated during the expiration. These excitatory neurons in PD also have a limited ability to slow breathing when activated during inspiration.

Respiratory phase-dependent function of inhibitory preBötC neurons in PD mice

Inhibitory preBötC neurons can promote increases or decreases in breathing frequency depending on their activity pattern (Baertsch et al., 2018). Inhibitory neurons active during the inspiratory phase promote faster breathing by limiting the duration of a refractory period that follows each inspiration. In contrast, inhibitory neurons with activity during the expiration delay the onset of inspiration resulting in slower breathing frequencies. To examine how these processes may be altered in PD mice, we generated mice that specifically express $\mathrm{ChR} 2{\text { in } \mathrm{Vgat}^{+}}^{+}$glycinergic and/or GABAergic neurons. Adult Vgat-ChR2 mice received injections of 6-OHDA or vehicle into the $\mathrm{CPu}$ followed by bilateral implantation of fiber optics targeting the preBötC. Bilateral fiber optics were placed in this group of mice because of the limited commissural projections of inhibitory (vs excitatory) preBötC neurons (Smith et al., 2013; Vann et al., 2018). As expected (Baertsch et al., 2018), in unanesthetized control mice, photostimulations during inspiration increased respiratory frequency by $27.2 \%$ (276.9 \pm 33.5 vs baseline: $217.7 \pm 14.1$ breaths/ min, two-way ANOVA, $\left.H_{(1,10)}=1.844, p=0.022\right)$; however, the same stimulations did not cause a significant frequency increase (16.1\%) in unanesthetized PD mice $(202.1 \pm 14.8$ vs baseline: $174.0 \pm 5.6$ breaths $/ \mathrm{min}$, two-way ANOVA, $F_{(1,10)}=1.716$, $p=0.221$; Fig. $7 A, B)$. Under anesthesia, when baseline $\mathrm{f}_{\mathrm{R}}$ is slow relative to unanesthetized conditions, photostimulation of $\mathrm{Vgat}^{+}$ preBötC neurons strongly increased breathing frequency in both control (132.4 \pm 2.4 vs baseline: $94.6 \pm 1.9$ breaths/min, two-way ANOVA, $\left.F_{(1,8)}=53.874, p<0.001\right)$ and PD mice $(136.3 \pm 13.1$ 


\section{Unanesthetized}

A
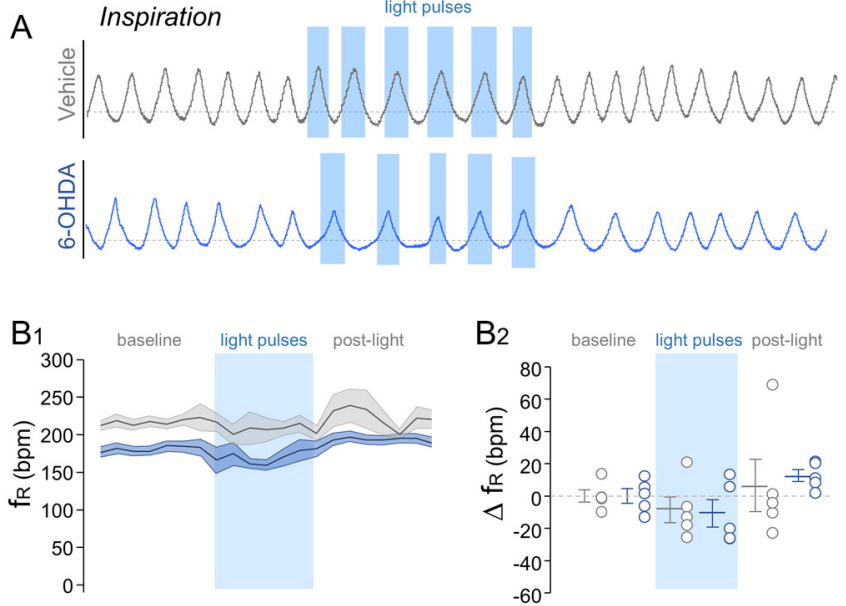

E

\section{Anesthetized}

$\frac{0}{0}$

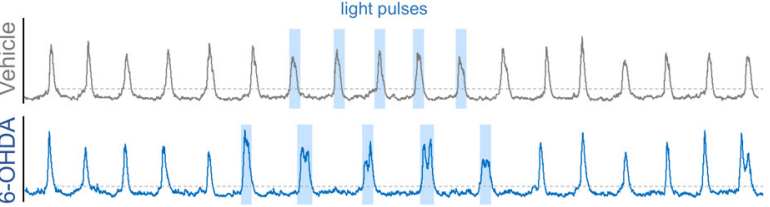

$\mathrm{F}_{1}$

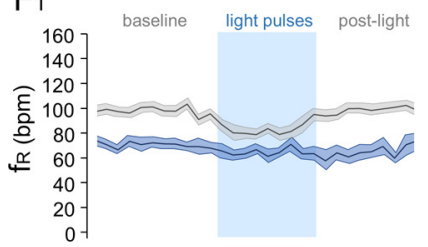

$\mathrm{F}_{2}$

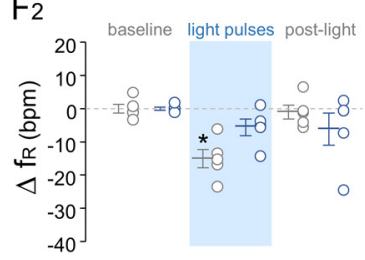

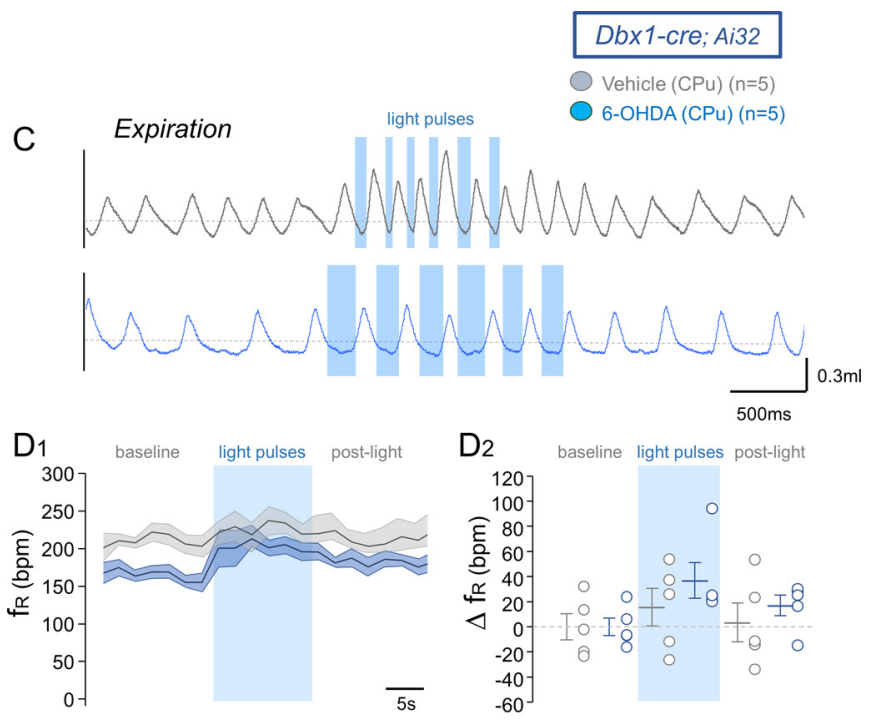

G

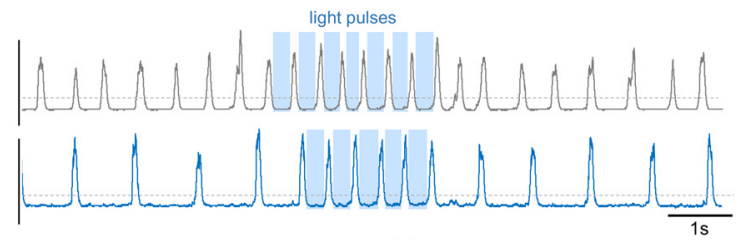

$\mathrm{H}_{1}$

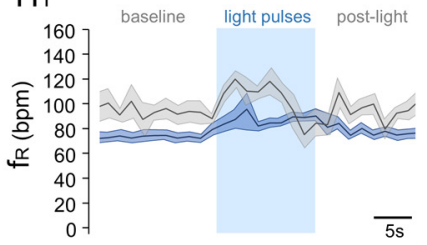

$\mathrm{H}_{2}$

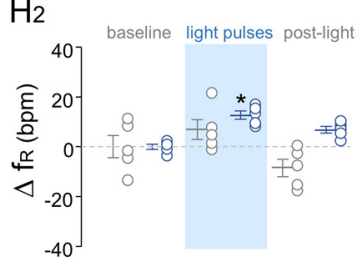

Figure 6. Dbx1 cells unilateral optogenetic activation changes breathing in control and PD mice model. $A, C$, Representative recordings from two Dbx $1^{+}$mice (vehicle-injected on the top and PD-induced mice on the bottom) during optogenetic activation during $(\boldsymbol{A})$ inspiration and $(\boldsymbol{C})$ expiration. Changes in $(\boldsymbol{B}) \mathrm{f}_{\mathrm{R}}$ at inspiration and $(\boldsymbol{D})$ expiration photostimulation in unanesthetized mice at room air 10-12 d after bilateral injection of 6-0HDA or vehicle into the (Pu ( $n=5 /$ group). B2, D2, 30-s recording average before, during, and 30-s average after photostimulation. $\boldsymbol{E}, \boldsymbol{G}$, Representative recordings from two anesthetized Dbx $1^{+}$mice (vehicle-injected on the top and PD-induced mice on the bottom) during optogenetic activation during $(\boldsymbol{E})$ inspiration and (G) expiration. Changes in $\mathrm{f}_{\mathrm{R}}$ of Dbx1-ChR2 animals during $(\boldsymbol{F})$ inspiration and $(\boldsymbol{H})$ expiration. $\boldsymbol{F} \mathbf{2}, \boldsymbol{H 2}, 20$-s recording average before, during, and 20-s average after photostimulation $(n=5 /$ group). Two-way ANOVA, followed by Newman-Keuls test, $p<0.05$.

vs baseline: $73.1 \pm 3.4$ breaths/min, two-way ANOVA, $F_{(1,8)}=$ $12.721, p=0.001$; Fig. $7 E, F)$, which represents a $\mathrm{f}_{\mathrm{R}}$ increase of $39.9 \%$ in control and $86.4 \%$ in PD-induced mice. When $\mathrm{Vgat}^{+}$ preBötC neurons were activated during the expiration, breathing frequency was dramatically slowed to a similar extent in control and PD mice under unanesthetized [control: $112.2 \pm 7.0$ vs baseline: $223.6 \pm 7.3$ breaths/min $(49.8 \%)$ and $\mathrm{PD}: 94.4 \pm 7.1$ vs baseline: $188.5 \pm 6.5$ breaths/min (49.9\%), two-way ANOVA, $F_{(1,10)}=45.47, p<0.001$; Fig. $7 C, D$ ] and anesthetized [control: $46.2 \pm 3.3$ vs baseline: $98.2 \pm 3.5$ breaths $/ \mathrm{min}(53 \%)$ and PD: $34.5 \pm 1.0$ vs baseline: $75.5 \pm 4.4$ breaths $/ \mathrm{min}$ (54.3\%), two-way ANOVA, $F_{(1,8)}=10.50, p=0.005$; Fig. $\left.7 G, H\right]$ conditions. Thus, consistent with our neuroanatomical results showing no changes in the number of inhibitory neurons within the VRC (Fig. 3E,F), our optogenetic experiments revealed no functional deficits in this neuronal population.

\section{Breathing frequency modulation by $\mathrm{PHOX}_{2}{ }^{+}$RTN neurons is preserved in PD mice}

Rhythm generating mechanisms in the preBötC are subject to strong modulatory influences to appropriately match breathing with the concurrent metabolic, environmental, or behavioral context. For example, PHOX2B-expressing neurons in the RTN are thought to be chemosensitive and provide excitatory drive to the preBötC in response to elevated $\mathrm{CO}_{2}$ (Takakura et al., 2006; Bochorishvili et al., 2012). Previous studies have described a reduction in PHOX2B RTN neurons (Tuppy et al., 2015; Fernandes-Junior et al., 2018) and an associate impairment in the hypercapnia ventilatory response (Tuppy et al., 2015; Oliveira et al., 2017). To explore whether this modulatory system may contribute to the respiratory impairments observed in our study (Fig. 2), we generated mice that express the ChR2 only in $\mathrm{PHOX}_{2} \mathrm{~B}^{+}$cells. Adult $\mathrm{PHOX} 2 \mathrm{~B}-\mathrm{ChR} 2$ mice were randomly selected for injections of 6-OHDA or vehicle into the $\mathrm{CPu}$, and an optical fiber was positioned at the level of the RTN unilaterally. Unfortunately, we were not able to identify phenotypically the PHOX2B neurons population in the photostimulated area and other PHOX2B neurons could also have been photostimulated, like A5 and C1 neurons. However, based on neuroanatomy, the optic fibers were positioned really close to the RTN/ pFRG most densely populated by PHOX2B neurons (Fig. 11) and consistent with recent studies (Abbott et al., 2009; Kanbar et al., 2010), plethysmography recordings confirmed that breathing frequency was potently increased by $33.8 \%$ in unanesthetized control mice $(262.6 \pm 16.4$ vs baseline: $196.2 \pm 6.6$ breaths $/ \mathrm{min}$, 


\section{Unanesthetized}

A
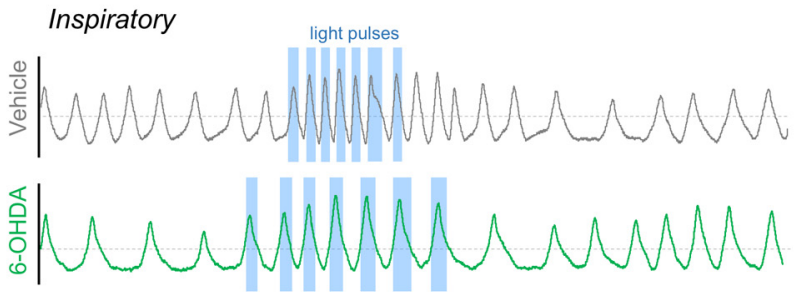

B 1

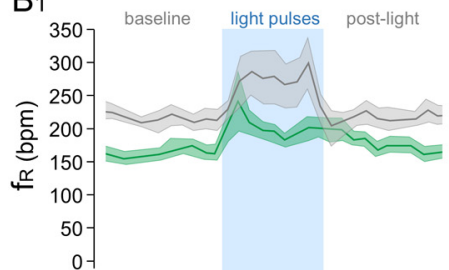

B2

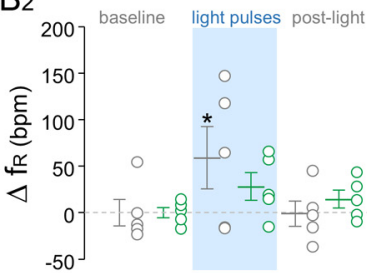

Anesthetized

E
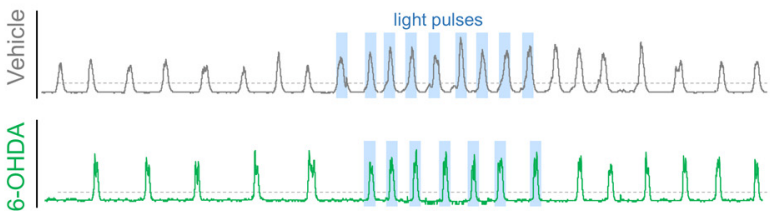

$\mathrm{F}_{1}$

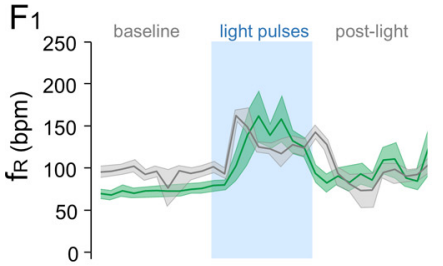

$\mathrm{F}_{2}$

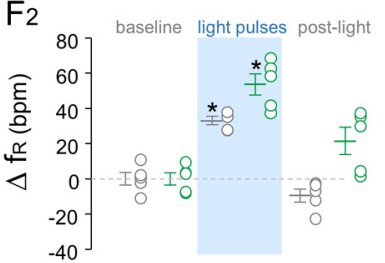

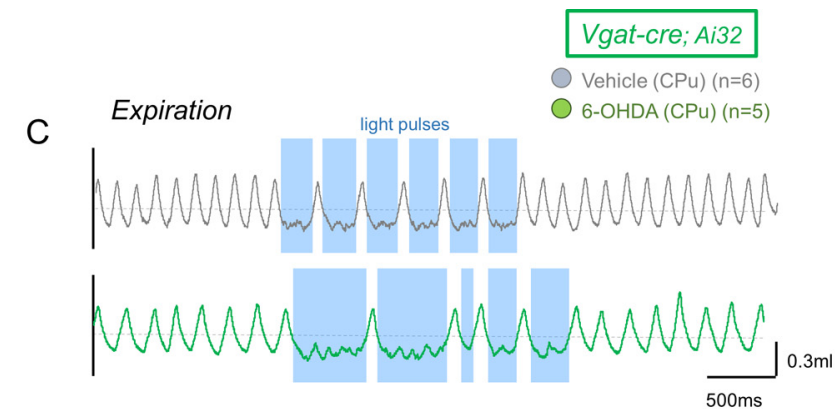

D1

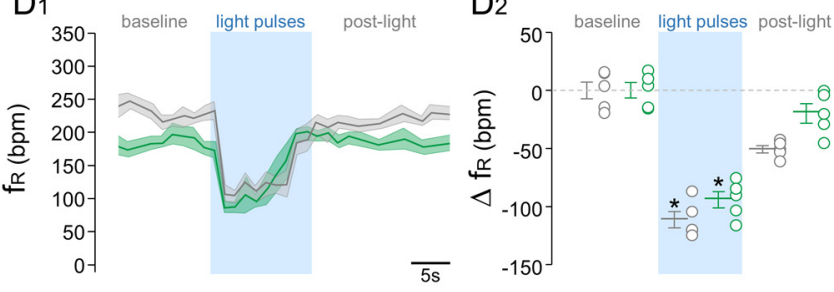

G
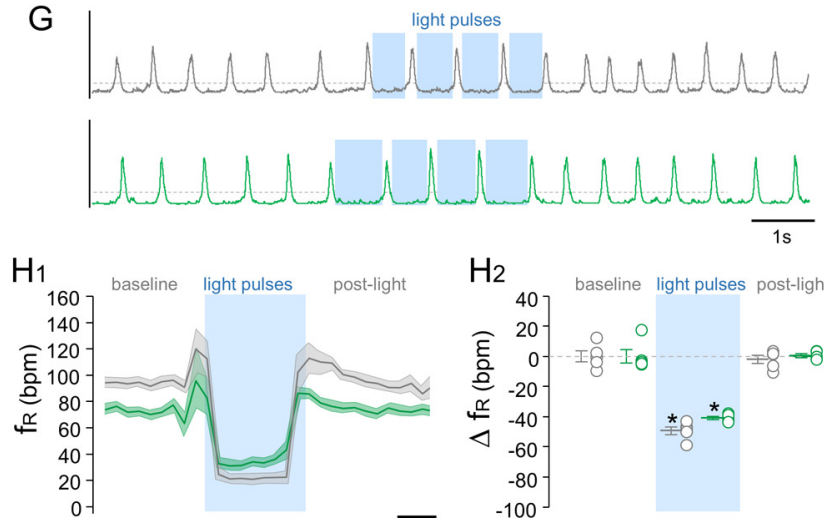

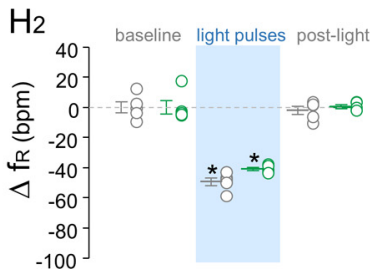

Figure 7. GABAergic cells bilateral optogenetic activation changes breathing in control and PD mice model. $A, C$, Representative recordings from two Vgat ${ }^{+}$mice (vehicle-injected on the top and PD-induced mice on the bottom) during optogenetic activation during $(\boldsymbol{A})$ inspiration and $(\boldsymbol{C})$ expiration. Changes in $(\boldsymbol{B}) \mathrm{f}_{\mathrm{R}}$ at inspiration and $(\boldsymbol{D})$ expiration photostimulation in unanesthetized mice at room air 10-12 d after bilateral injection of 6-0HDA or vehicle into the (Pu ( $n=5-6 /$ group). B2, D2, 30-s recording average before, during, and 30-s average after photostimulation. $\boldsymbol{E}, \boldsymbol{G}$, Representative recordings from two anesthetized $\mathrm{Vgat}^{+}$mice (vehicle-injected on the top and PD-induced mice on the bottom) during optogenetic activation during $(\boldsymbol{E}$ ) inspiration and $(\boldsymbol{G})$ expiration. Changes in $\mathrm{f}_{\mathrm{R}}$ of $\mathrm{Vgat}$-ChR2 animals during $(\boldsymbol{F})$ inspiration and $(\boldsymbol{H})$ expiration. $\boldsymbol{F 2}, \mathbf{H 2}$, 20-s recording average before, during, and 20-s average after photostimulation ( $n=5-6 /$ group). Two-way ANOVA, followed by Newman-Keuls test, $p<0.05$.

\section{Unanesthetized}

A

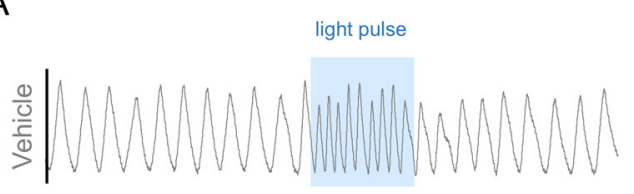

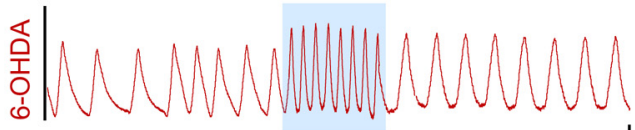

B

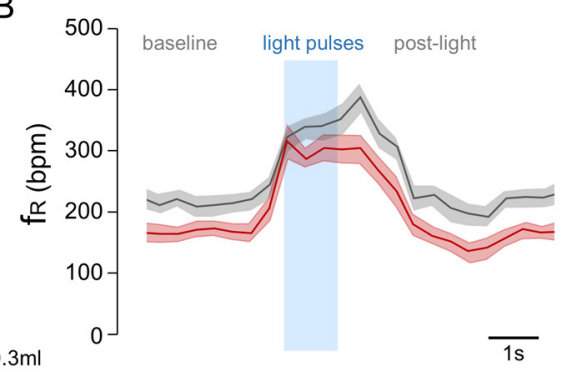

\section{PHOX2B-cre; Ai32}

Vehicle $(\mathrm{CPu})(\mathrm{n}=8)$

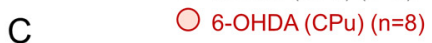

C

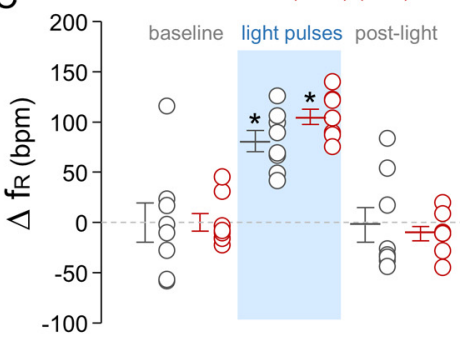

Figure 8. $\mathrm{PHOX} 2 \mathrm{~B}^{+}$cells unilateral optogenetic activation changes breathing in control and PD mice model. $A$, Representative recordings from two PHOX2B ${ }^{+}$mice (vehicle-injected on the top and PD-induced mice on the bottom) during optogenetic activation for $1 \mathrm{~s}$ continuous. Changes in $(\boldsymbol{B}) \mathrm{f}_{\mathrm{R}}$ in unanesthetized mice at room air 10-12 $\mathrm{d}$ after bilateral injection of 6-0HDA or vehicle into the CPu. C, 30-s recording average before, during (1 s), and 30-s average after photostimulation normalized data from vehicle-injected and 6-OHDA-injected. Two-way ANOVA, followed by Newman-Keuls test, $p<0.05$. 


\section{Inspiration}

Threshold set to the very onset of inspiration $\int_{V} f_{R}$

A Vglut2-cre; $A$ i3
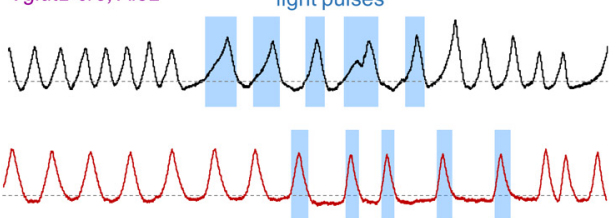

C Dbx1-cre; Ai32
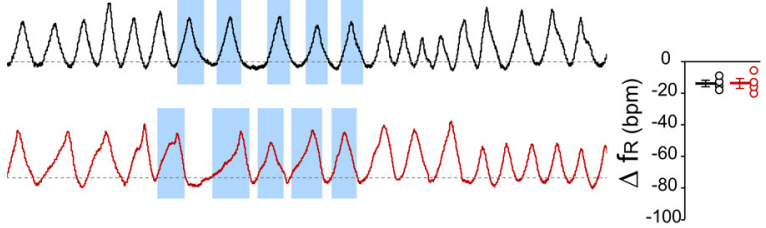

E

Vglut2-cre; Ai32 light pulses

WNANMNMNANMNAN

个f $f_{R}$

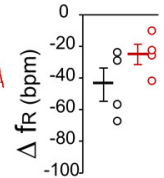

$M A M A M M M$
Threshold set late in inspiration

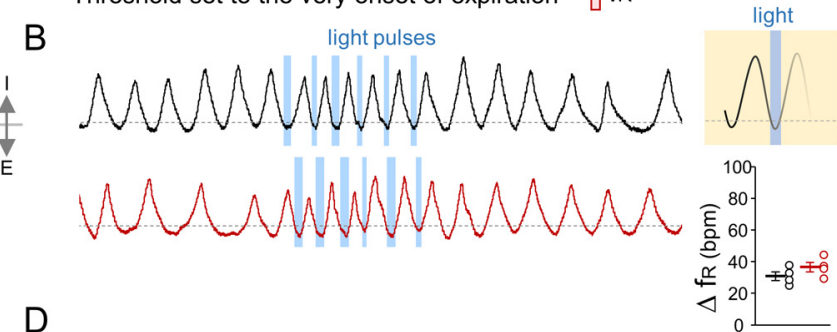

A A A AHAAHANAAAA

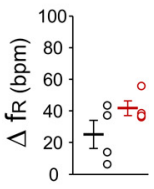

G

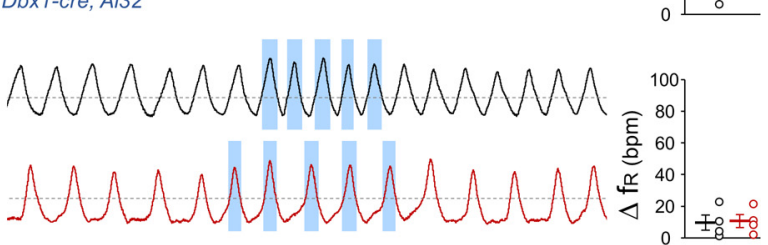

Expiration

Threshold set to the very onset of expiration $\widehat{\jmath} f_{R}$

mublum

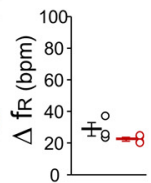

$\mathrm{F}$

Threshold set late in expiration
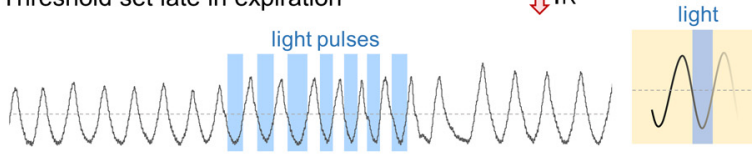

O Vehicle $(\mathrm{CPu})(\mathrm{n}=4-5)$

6-OHDA (CPu) $(n=3-4)$
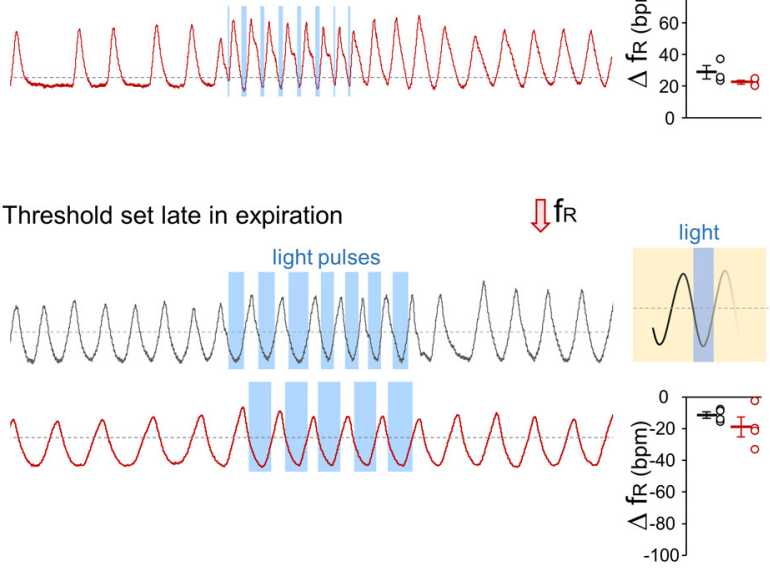

$\mathrm{H}$

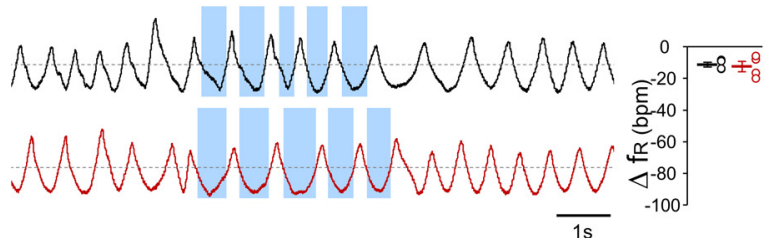

Figure 9. Threshold differently positioned produces variable responses on breathing. $\mathbf{A - D}$, Recordings from $\mathrm{Vglut}_{2}{ }^{+}$or $\mathrm{Dbx}^{+}{ }^{+}$mice (vehicle-injected on the top and PD-induced mice on the bottom) representing the threshold set to the very onset of inspiration $(\boldsymbol{A}, \boldsymbol{C})$ or expiration $(\boldsymbol{B}, \boldsymbol{D})$ which produce the effects observed in the regular Figures 3,4 . $\boldsymbol{E}-\boldsymbol{H}$, Excluded data groups, because the threshold was set late in inspiration $(\boldsymbol{E}, \boldsymbol{G})$ or expiration $(\boldsymbol{F}, \boldsymbol{H})$, which produced unexpected ventilatory responses along the experiments. On the right of each representative recording, it is the average of $f_{R}$ at photostimulation period normalized by before.

two-way ANOVA, $\left.F_{(1,17)}=20.963, p<0.001\right)$ during a 1 -s continuous optogenetic activation of PHOX2B neurons in the RTN. A robust respiratory response was observed in PD mice $(62.9 \%$; $248.6 \pm 5.8$ vs baseline: $152.6 \pm 4.0$ breaths/min; Fig. 8 ), suggesting that changes in the modulatory influence of putative chemosensitive RTN neurons do not underlie the respiratory impairments observed in the PD mouse model used here.

\section{Discussion}

The primary goal of our study was to characterize the cell-typespecific anatomic and functional deficits that underlie respiratory impairments associated with PD. We found that putatively rhythmically active glutamatergic-Dbxl neurons in the VRC selectively has been reduced in a mouse model of PD. These neurons may be particularly susceptible to be reduced in the context of PD since inhibitory VRC neurons, as well as neurons in other respiratory-related regions, do not undergo a similar neuronal reduction. Consistent with our neuroanatomical findings, optogenetic experiments revealed that changes in PD mice were specific to manipulations involving glutamatergic Dbx1 neurons. Thus, we present the hypothesis that breathing abnormalities in
PD are caused by the specific decreased number of this critical population of respiratory neurons.

\section{Respiratory consequences of a decreased number of glutamatergic Dbx1 neuron}

The inspiratory rhythm emerges as a consequence of the periodic synchronization of neuronal activity within the preBötC. This region and the adjacent $\mathrm{VRC}$ is comprised of heterogeneous populations of excitatory and inhibitory neurons. However, despite the important role of inhibition for promoting the dynamic control of inspiration (Baertsch et al., 2018; Baertsch and Ramirez, 2019), inhibitory mechanisms are not necessary for rhythmogenesis per se (Janczewski et al., 2013). Instead, the "Dbx1 core hypothesis" posits that a subpopulation of glutamatergic neurons derived from precursors that express the transcription factor Dbx1 are critical for generating the inspiratory rhythm (Vann et al., 2016, 2018). Mice born with a genetic deletion of Dbx1 do not exhibit inspiratory efforts and die shortly after birth (Gray et al., 2010). Consistent with these initial observations, acute progressive ablation of relatively small numbers $(\sim 15 \%)$ of Dbx1 neurons in brainstem slices containing the 
Unanesthetized

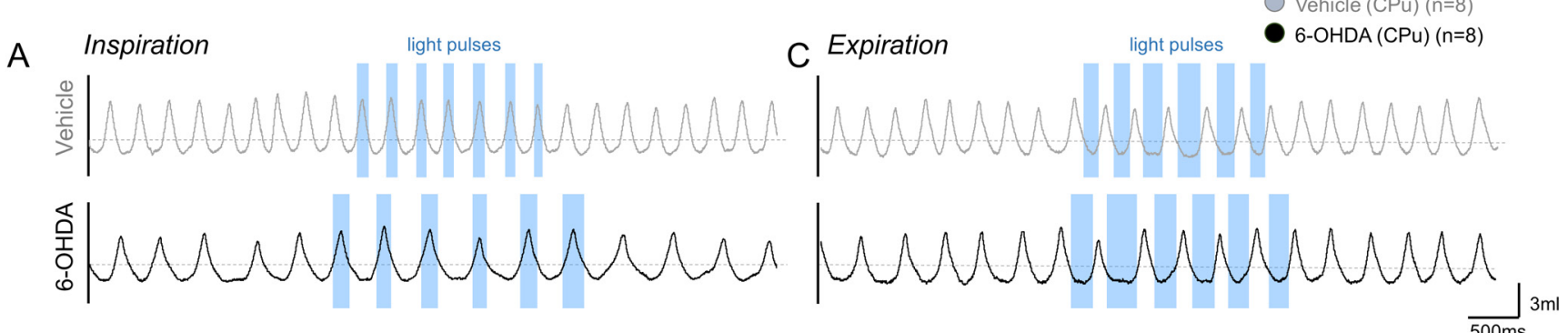

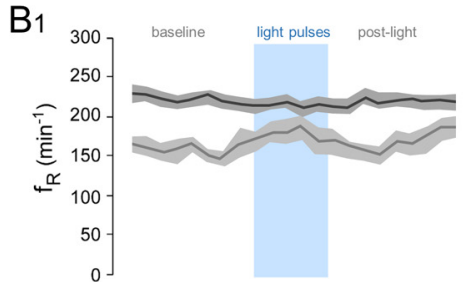

Anesthetized

$\mathrm{E}$
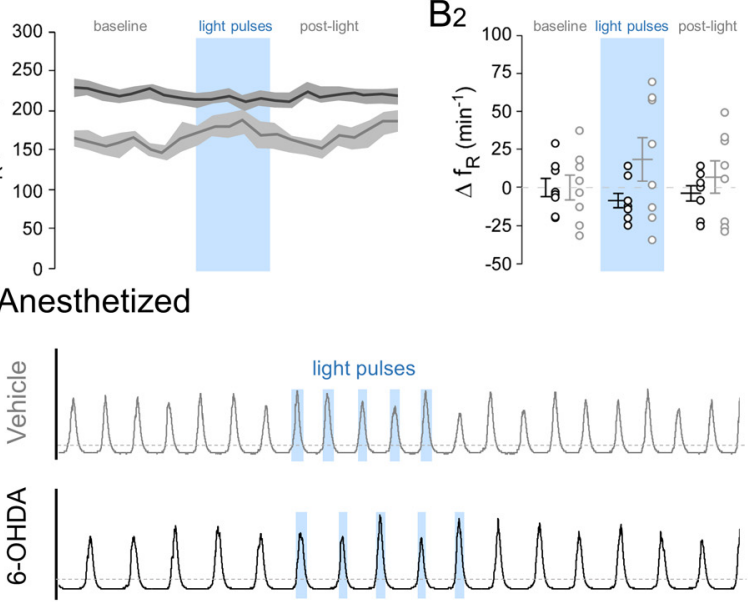

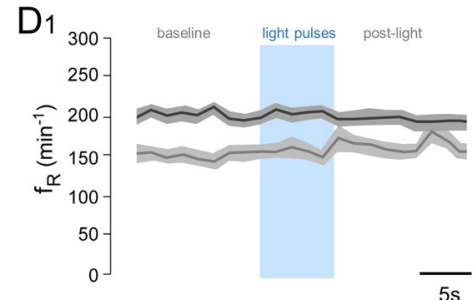

$\mathrm{D} 2$

G
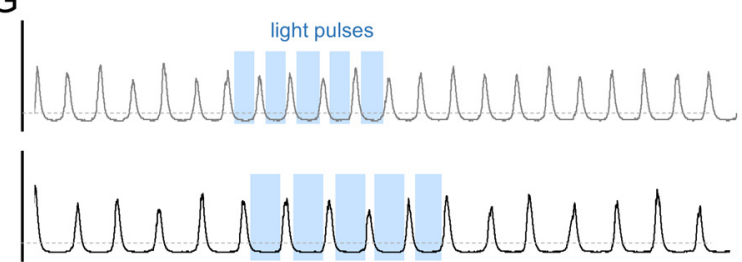
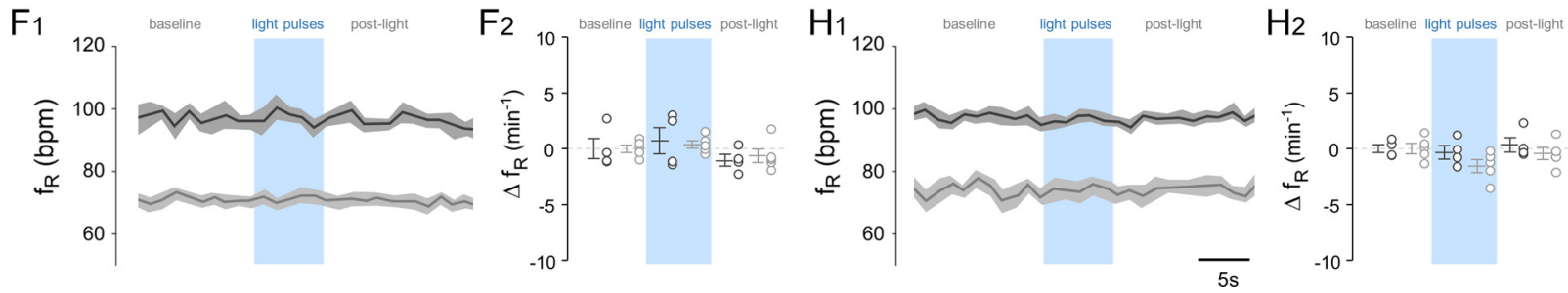

Figure 10. Cre ${ }^{-}$cells unilateral optogenetic activation do not change breathing in control and PD mice. $A, C$, Representative recordings from two cre ${ }^{-}$mice (vehicle-injected on the top and $\mathrm{PD}$-induced mice on the bottom) during optogenetic activation during $(\boldsymbol{A})$ inspiration and $(\boldsymbol{C})$ expiration phase. Changes in $(\boldsymbol{B}) \mathrm{f}_{\mathrm{R}}$ at inspiration and $(\boldsymbol{D})$ expiration phase photostimulation in unanesthetized mice at room air 10-12 d after bilateral injection of 6-0HDA or vehicle into the (Pu ( $n=5-6 /$ group). B2, D2, 30-s recording average before, during, and 30-s average after photostimulation. Representative recordings from $\boldsymbol{E}, \boldsymbol{G}$, two anesthetized cre ${ }^{-}$mice (vehicle-injected on the top and PD-induced mice on the bottom) during optogenetic activation during $(\boldsymbol{E})$ inspiration and $(\boldsymbol{G})$ expiration phase. Changes in $\mathrm{f}_{\mathrm{R}}$ of $\mathrm{cre}^{-}$animals during $(\boldsymbol{F})$ inspiration and $(\boldsymbol{H})$ expiration. $\boldsymbol{F 2}, \boldsymbol{H 2}$, 20-s recording average before, during, and 20-s average after photostimulation ( $n=8$ /group).

preBötC causes the inspiratory rhythm to slow down, become more irregular, and eventually stop altogether (Wang et al., 2014). Furthermore, in model preBötC networks that only contain excitatory neurons, the frequency and regularity of the rhythm depends on the number of excitatory neurons within the network as well as the connectivity between them (Carroll and Ramirez, 2013). Based on experimental and computational studies, this excitatory connectivity seems to be fairly sparse (Carroll and Ramirez, 2013). Indeed, sparse excitatory interactions seems to be the critical anatomic substrate that allows the preBötC network to synchronize. On the other hand, synchrony among excitatory neurons is weakened by inhibitory neurons within the network (Baertsch et al., 2018), and computational models suggest that in networks with too much inhibition, the rhythm falls apart (Harris et al., 2017). Under normal conditions, this balance between excitation and inhibition seems to be regulated such that the network operates on the "edge" of synchrony, allowing the rhythm to be highly flexible (Baertsch et al., 2018;
Baertsch and Ramirez, 2019), but also making it particularly susceptible to abnormal changes in the function of excitatory neurons (Wang et al., 2014; Harris et al., 2017).

In this study, we found a decreased number of glutamatergic Dbx1 neurons in a mouse model of PD. When integrated within the wider medullary network, there is evidence that the anatomic and functional boundaries of the preBötC are spatially and dynamically regulated (Baertsch et al., 2019). Therefore, we did not attempt to localize a preBötC "kernel" but instead examined neuroanatomical changes along the rostrocaudal extent of the VRC, which includes the preBötC. Because not all glutamatergic $\left(\mathrm{Vglut}_{2}{ }^{+}\right)$neurons in the VRC are derived from Dbx1 expressing cells, and Dbx1-expressing progenitors can differentiate into both neurons and glia (Kottick et al., 2017), we employed both genetic labeling strategies to gain insights into anatomic changes involving specifically glutamatergic Dbx1-derived neurons. Cell counting revealed $\sim 1600$ fewer $(-17 \%)$ Vglut $_{2}{ }^{+}$neurons in the VRC of PD mice compared with healthy controls (Fig. 3). Similarly, there were $\sim 950$ fewer $(-47 \%)$ Dbxl cells (Fig. 3), 

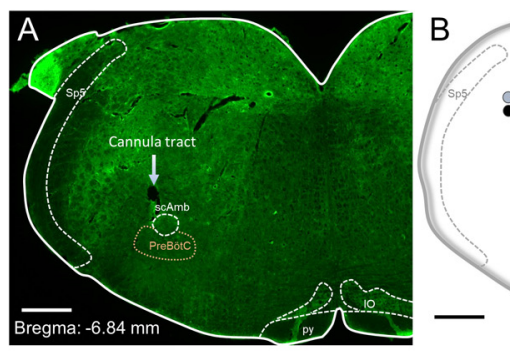

D

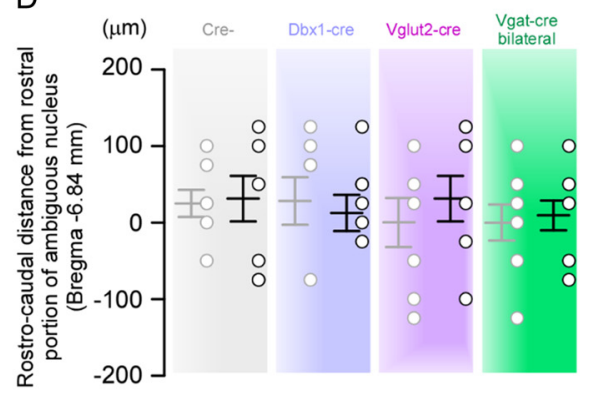

G

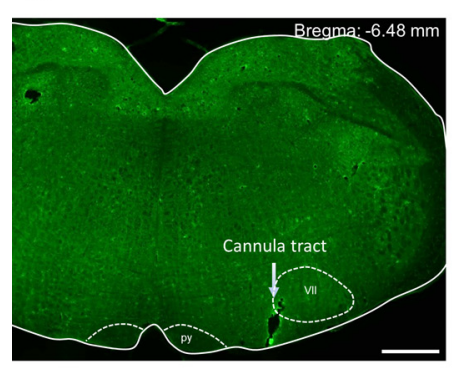

$\mathrm{H}$

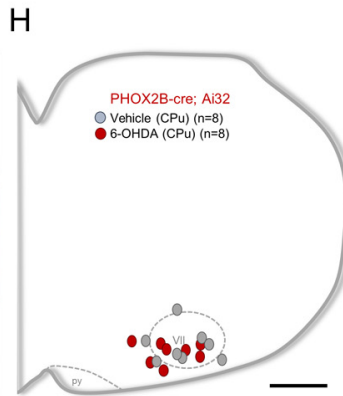

E
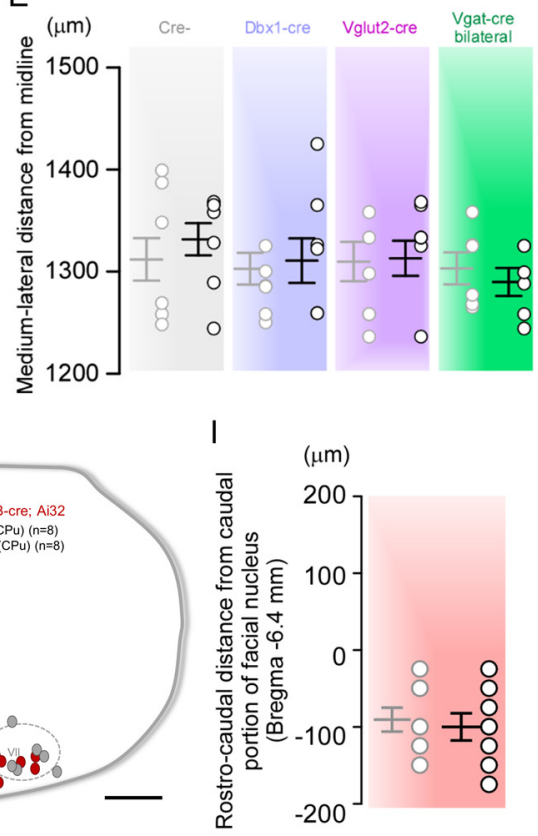

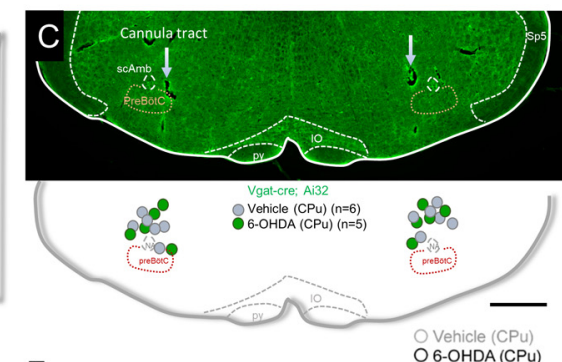

$\mathrm{F}$
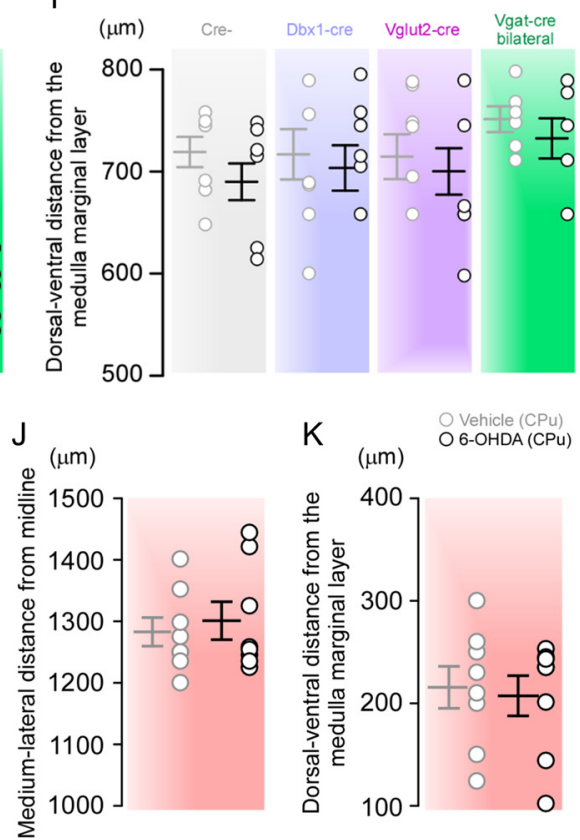

Figure 11. Fiber optics tracts used in our study. $\boldsymbol{A}$, Photomicrograph showing typical unilateral fiber optics tracts in the preBötC region. $\boldsymbol{B}$, Computer-generated plot of fiber optics placement that were confine to the preBötC region (bregma $-6.84 \mathrm{~mm}$ according to the Paxinos and Franklin Atlas). $\boldsymbol{C}$, Photomicrograph showing typical bilateral fiber optics and computer-generated plot in the preBötC region. $\boldsymbol{D}$, Rostro-caudal distance from ambiguus nucleus (bregma $-6.84 \mathrm{~mm}$ ). $\boldsymbol{E}$, Lateral distance from midline. $\boldsymbol{F}$, Dorsal distance from ventral marginal layer ( $\mu$ m). $\boldsymbol{G}$, Photomicrograph showing typical unilateral fiber optics tracts and $(\boldsymbol{H})$ computer-generated plot of fiber optics placement in the RTN region. $\boldsymbol{I}$, Rostro-caudal distance from caudal portion of facial nucleus (bregma $-6.4 \mathrm{~mm}$ ). $\boldsymbol{J}$, Lateral distance from midline. $\boldsymbol{K}$, Dorsal distance from ventral marginal layer ( $\mu \mathrm{m})$. scAmb, ambiguus nucleus semi-compact pars; I0, inferior olives; py, pyramids; Sp5, spinal trigeminal tract; VIl, facial nucleus; preBötC, pre-Bötzinger complex. Scale bars: $250 \mu \mathrm{m}(\boldsymbol{A}, \boldsymbol{B}), 400 \mu \mathrm{m}(\boldsymbol{C})$, and $600 \mu \mathrm{m}(\boldsymbol{G}, \boldsymbol{H})$.

which was predominantly driven by a reduction in the number of Dbx1 neurons rather than Dbx1 astrocytes (Fig. 3).

In contrast, the number of glycinergic and/or GABAergic Vgat-expressing neurons in the VRC did not change in PD mice, suggesting these inhibitory neurons do not undergo a similar neuronal loss. Thus, in the context of PD, we hypothesize that the balance of excitation and inhibition is altered within the VRC causing rhythmogenesis to become unstable and leading to the slow irregular breathing patterns observed in PD mice (Fig. 2; Tuppy et al., 2015; Oliveira et al., 2017).

\section{The three phases of the inspiratory rhythm, implications in PD mice}

Breathing abnormalities in PD mice were because of longer, more irregular intervals between inspiratory efforts, i.e., the expiratory time (Fig. 2). This is typical for alterations in breathing frequency; however, the underlying mechanisms regulating respiratory frequency are complex. Within the inspiratory network, the rhythm can be broken down into three sequential, mechanistically distinct, phases that differentially regulate inspiratory frequency: the percolation phase, burst phase, and refractory phase (Kottick and Del Negro, 2015; Cui et al., 2016; Cregg et al., 2017; Baertsch et al., 2018). During the percolation phase, it is thought that synaptically and intrinsically driven spiking activity begins to propagate among interconnected excitatory neurons (Cui et al., 2016). This stochastic feed-forward process (Carroll and Ramirez, 2013) progressively increases excitability in the network until voltage-dependent and $\mathrm{Ca}^{2+}$-dependent membrane conductances are activated leading to a rapid depolarization and increased spiking during the burst phase. During each burst, activity-dependent mechanisms are activated that cause a transient decrease in network activity that is maximal on burst termination. As this refractory phase subsides, percolation begins to build network excitability, and the cycle restarts.

As a consequence of these different rhythmogenic phases, manipulating the activity excitatory or inhibitory preBötC neurons has phase-dependent effects on breathing frequency (Sherman et al., 2015; Cregg et al., 2017; Baertsch et al., 2018; Vann et al., 2018). Increasing the activity of excitatory neurons during the burst phase lengthens the refractory phase, delaying the next burst, whereas increasing their activity during the expiration shortens the percolation phase, accelerating the onset of the next burst. Activating inhibitory neurons has the opposite effects, i.e., activation during the burst phase shortens the refractory phase, reducing the time required for the next burst occurs, whereas activating them during the expiration slows percolation, delaying or preventing the onset of the next burst. 
In the current study, we explored the phase-dependent regulation of breathing frequency in a mouse model of PD. We examined not only anesthetized, but also unanesthetized mice, and although baseline breathing frequency is $\sim 2 \times$ faster and highly modulated by behavior in unanesthetized mice, our results were generally consistent between the two experimental preparations. Interestingly, in PD mice, increasing the activity of glutamatergic Vglut $_{2}{ }^{+}$neurons during the burst phase did not significantly slow breathing as expected (Fig. $5 A, B, E, F$ ), suggesting the refractory phase may not regulate the duration of the expiration as strongly in PD mice. This may be explained by the significantly reduced number of excitatory neurons in PD. Indeed, when the activity of $\mathrm{Vglut}_{2}{ }^{+}$neurons was increased during the expiration, breathing frequency increased to similar levels in PD and control mice (Fig. 5C,D,G,H), indicating that enhancing specifically the percolation phase can restore breathing in PD mice. Although trends were similar when activating Dbxl neurons, the effects were generally weaker (Baertsch et al., 2018; Vann et al., 2018), which is likely because of fewer total neurons and innervating terminals activated in the region. Thus, the optogenetic stimulation experiments presented here allow us to make very specific predictions. We specifically hypothesize that the expiratory time is prolonged in $\mathrm{PD}$ mice because of a slower percolation phase caused by fewer interconnected excitatory neurons participating in the inspiratory rhythm. Since the neuropeptide substance P has been shown to increase breathing frequency and also reduce irregularity by enhancing the percolation phase (Baertsch and Ramirez, 2019), we predict that the breathing frequency irregularities observed in PD mice are also caused by a weakened percolation phase.

Brainstem microcircuits (i.e., KF, PiCo, and others) seem to be a key source of inputs (excitatory or inhibitory) that drive phase transitions between inspiration and postinspiration (Dutschmann and Herbert, 2006; Anderson et al., 2016; Geerling et al., 2017). Here, we found a decreased number of KF glutamatergic neurons and PiCo cholinergic neurons in PD mice. The integrity of those nuclei are important for many behavioral movements of the upper airway such as cough, swallowing, speech and protective reflexes (Fregosi and Ludlow, 2014; Anderson et al., 2016; Toor et al., 2019). Discoordination of these neuromuscular activities may be related to several symptoms typically observed in PD patients (Simons, 2017), and should be a focus of future investigations.

A leading cause of morbidity and mortality in PD can be attributed to respiratory control and coordination abnormalities. Thus, studies aimed at improving our understanding of the specific neurophysiological mechanisms that underlie these breathing abnormalities will be key to improving survival and quality of life in PD patients. Although no animal model is without caveats, the 6-OHDA mouse model of PD used here and in several prior studies (Tuppy et al., 2015; Andrzejewski et al., 2017; Fernandes-Junior et al., 2018; Oliveira et al., 2019), reproduces breathing abnormalities and provides a means to rigorously investigate them in great detail. There is no doubt that different experimental models should also be tested. However, the anatomic and functional characterization performed in this study suggests that a decreased number of a specific subgroup of rhythmically active glutamatergic neurons in the ventral medulla may contribute significantly to respiratory complications in PD. Strategies that modulate specific phases of the inspiratory cycle may best address the breathing abnormalities in PD. Such strategies may be pharmacological (Wang et al., 2014; Kottick and Del Negro, 2015; Vann et al., 2018; Baertsch and Ramirez, 2019), or electrical/optical manipulations that can be artificially modulated for phase-dependence (Feldman et al., 2013; Anderson et al., 2016; Cui et al., 2016; Baertsch et al., 2018; Vann et al., 2018). Indeed, this study only represents a first step as future investigations aimed at developing approaches to strengthen excitatory interactions within the preBötC will be important as we move toward treatments for breathing abnormalities associated with PD.

\section{References}

Abbott SB, Stornetta RL, Fortuna MG, Depuy SD, West GH, Harris TE, Guyenet PG (2009) Photostimulation of retrotrapezoid nucleus phox2bexpressing neurons in vivo produces long-lasting activation of breathing in rats. J Neurosci 29:5806-5819.

Akins VT, Weragalaarachchi K, Picardo MCD, Revill AL, Del Negro CA (2017) Morphology of Dbx1 respiratory neurons in the preBötzinger complex and reticular formation of neonatal mice. Sci Data 4:170097.

Anderson TM, Garcia AJ, Baertsch NA, Pollak J, Bloom JC, Wei AD, Rai KG, Ramirez JM (2016) A novel excitatory network for the control of breathing. Nature 536:76-80.

Andrzejewski K, Budzińska K, Kaczyńska K (2017) Phrenic and hypoglossal nerve activity during respiratory response to hypoxia in 6-OHDA unilateral model of Parkinson's disease. Life Sci 180:143-150.

Angel RW, Alston W, Higgins JR (1970) Control of movement in Parkinson's disease. Brain 93:1-14.

Baertsch NA, Ramirez JM (2019) Insights into the dynamic control of breathing revealed through cell-type-specific responses to substance P. Elife 8: e51350.

Baertsch NA, Baertsch HC, Ramirez JM (2018) The interdependence of excitation and inhibition for the control of dynamic breathing rhythms. Nat Commun 9:843.

Baertsch NA, Severs LJ, Anderson TM, Ramirez JM (2019) A spatially dynamic network underlies the generation of inspiratory behaviors. Proc Natl Acad Sci USA 116:7493-7502.

Baille G, De Jesus AM, Perez T, Devos D, Dujardin K, Charley CM, Defebvre L, Moreau C (2016) Ventilatory dysfunction in Parkinson's disease. J Parkinsons Dis 6:463-471.

Bochorishvili G, Stornetta RL, Coates MB, Guyenet PG (2012) Pre-Bötzinger complex receives glutamatergic innervation from galaninergic and other retrotrapezoid nucleus neurons. J Comp Neurol 520:1047-1061.

Carroll MS, Ramirez JM (2013) Cycle-by-cycle assembly of respiratory network activity is dynamic and stochastic. J Neurophysiol 109:296-305.

Cregg JM, Chu KA, Dick TE, Landmesser LT, Silver J (2017) Phasic inhibition as a mechanism for generation of rapid respiratory rhythms. Proc Natl Acad Sci USA 114:12815-12820.

Cui Y, Kam K, Sherman D, Janczewski WA, Zheng Y, Feldman JL (2016) Defining preBötzinger complex rhythm- and pattern-generating neural microcircuits in vivo. Neuron 91:602-614.

Depocas F, Hart JS (1957) Use of the Pauling oxygen analyzer for measurement of oxygen consumption of animals in open-circuit systems and in a short-lag, closed-circuit apparatus. J Appl Physiol 10:388-392.

Dutschmann M, Herbert H (2006) The Kölliker-Fuse nucleus gates the postinspiratory phase of the respiratory cycle to control inspiratory off-switch and upper airway resistance in rat. Eur J Neurosci 24:1071-1084.

Dutschmann M, Bautista TG, Trevizan-Baú P, Dhingra RR, Furuya WI (2021) The pontine Kölliker-Fuse nucleus gates facial, hypoglossal, and vagal upper airway related motor activity. Respir Physiol Neurobiol 284:103563.

Feldman JL, Del Negro CA, Gray PA (2013) Understanding the rhythm of breathing: so near, yet so far. Annu Rev Physiol 75:423-452.

Fernandes-Junior SA, Carvalho KS, Moreira TS, Takakura AC (2018) Correlation between neuroanatomical and functional respiratory changes observed in an experimental model of Parkinson's disease. Exp Physiol 103:1377-1389.

Fregosi RF, Ludlow CL (2014) Activation of upper airway muscles during breathing and swallowing. J Appl Physiol (1985) 116:291-301.

Geerling JC, Yokota S, Rukhadze I, Roe D, Chamberlin NL (2017) KöllikerFuse GABAergic and glutamatergic neurons project to distinct targets. J Comp Neurol 525:1844-1860.

Goes ATR, Jesse CR, Antunes MS, Lobo Ladd FV, Lobo Ladd AAB, Luchese C, Paroul N, Boeira SP (2018) Protective role of chrysin on 6-hydroxydopamine-induced neurodegeneration a mouse model of Parkinson's 
disease: involvement of neuroinflammation and neurotrophins. Chem Biol Interact 279:111-120.

Gray PA, Hayes JA, Ling GY, Llona I, Tupal S, Picardo MC, Ross SE, Hirata T, Corbin JG, Eugenín J, Del Negro CA (2010) Developmental origin of preBötzinger complex respiratory neurons. J Neurosci 30:14883-14895.

Harris KD, Dashevskiy T, Mendoza J, Garcia AJ, Ramirez JM, Shea-Brown E (2017) Different roles for inhibition in the rhythm-generating respiratory network. J Neurophysiol 118:2070-2088.

Hayes JA, Kottick A, Picardo MCD, Halleran AD, Smith RD, Smith GD, Saha MS, Del Negro CA (2017) Transcriptome of neonatal preBötzinger complex neurones in Dbx1 reporter mice. Sci Rep 7:8669.

Huckstepp RT, Cardoza KP, Henderson LE, Feldman JL (2015) Role of parafacial nuclei in control of breathing in adult rats. J Neurosci 35:1052-1067.

Janczewski WA, Tashima A, Hsu P, Cui Y, Feldman JL (2013) Role of inhibition in respiratory pattern generation. J Neurosci 33:5454-5465.

Kam K, Worrell JW, Janczewski WA, Cui Y, Feldman JL (2013) Distinct inspiratory rhythm and pattern generating mechanisms in the preBötzinger complex. J Neurosci 33:9235-9245.

Kanbar R, Stornetta RL, Cash DR, Lewis SJ, Guyenet PG (2010) Photostimulation of Phox $2 \mathrm{~b}$ medullary neurons activates cardiorespiratory function in conscious rats. Am J Respir Crit Care Med 182:1184-1194.

Kottick A, Del Negro CA (2015) Synaptic depression influences inspiratoryexpiratory phase transition in Dbxl interneurons of the preBötzinger complex in neonatal mice. J Neurosci 35:11606-11611.

Kottick A, Martin CA, Del Negro CA (2017) Fate mapping neurons and glia derived from Dbx1-expressing progenitors in mouse preBötzinger complex. Physiol Rep 5:e13300.

Lazarenko RM, Milner TA, Depuy SD, Stornetta RL, West GH, Kievits JA, Bayliss DA, Guyenet PG (2009) Acid sensitivity and ultrastructure of the retrotrapezoid nucleus in Phox2b-EGFP transgenic mice. J Comp Neurol 517:69-86.

Maria B, Sophia S, Michalis M, Charalampos L, Andreas P, John ME, Nikolaos SM (2003) Sleep breathing disorders in patients with idiopathic Parkinson's disease. Respir Med 97:1151-1157.

Marina N, Abdala AP, Trapp S, Li A, Nattie EE, Hewinson J, Smith JC, Paton JF, Gourine AV (2010) Essential role of Phox 2b-expressing ventrolateral brainstem neurons in the chemosensory control of inspiration and expiration. J Neurosci 30:12466-12473.

Moreno Catalá M, Woitalla D, Arampatzis A (2016) Reactive but not predictive locomotor adaptability is impaired in young Parkinson's disease patients. Gait Posture 48:177-182.

Mulkey DK, Stornetta RL, Weston MC, Simmons JR, Parker A, Bayliss DA, Guyenet PG (2004) Respiratory control by ventral surface chemoreceptor neurons in rats. Nat Neurosci 7:1360-1369.

Oliveira LM, Tuppy M, Moreira TS, Takakura AC (2017) Role of the locus coeruleus catecholaminergic neurons in the chemosensory control of breathing in a Parkinson's disease model. Exp Neurol 293:172-180.

Oliveira LM, Oliveira MA, Moriya HT, Moreira TS, Takakura AC (2019) Respiratory disturbances in a mouse model of Parkinson's disease. Exp Physiol 104:729-739.

Owolabi LF, Nagoda M, Babashani M (2016) Pulmonary function tests in patients with Parkinson's disease: a case-control study. Niger J Clin Pract 19:66-70.

Patrone LGA, Biancardi V, Marques DA, Bícego KC, Gargaglioni LH (2018) Brainstem catecholaminergic neurones and breathing control during postnatal development in male and female rats. J Physiol 596:3299-3325.

Paxinos G, Franklin K (2012) The mouse brain in estereotaxic coordinates, Ed 1. San Diego: Elsevier.

Schiffer BL, Kendall K (2019) Changes in timing of swallow events in Parkinson's disease. Ann Otol Rhinol Laryngol 128:22-27.

Seccombe LM, Giddings HL, Rogers PG, Corbett AJ, Hayes MW, Peters MJ, Veitch EM (2011) Abnormal ventilatory control in Parkinson's disease- further evidence for non-motor dysfunction. Respir Physiol Neurobiol 179:300-304.

SheikhBahaei S, Morris B, Collina J, Anjum S, Znati S, Gamarra J, Zhang R, Gourine AV, Smith JC (2018) Morphometric analysis of astrocytes in brainstem respiratory regions. J Comp Neurol 526:2032-2047.

Sherman D, Worrell JW, Cui Y, Feldman JL (2015) Optogenetic perturbation of preBötzinger complex inhibitory neurons modulates respiratory pattern. Nat Neurosci 18:408-414.

Simons JA (2017) Swallowing dysfunctions in Parkinson's disease. Int Rev Neurobiol 134:1207-1238.

Smith DV, Li CS, Davis BJ (1998) Excitatory and inhibitory modulation of taste responses in the hamster brainstem. Ann NY Acad Sci 855:450-456.

Smith JC, Ellenberger HH, Ballanyi K, Richter DW, Feldman JL (1991) PreBötzinger complex: a brainstem region that may generate respiratory rhythm in mammals. Science 254:726-729.

Smith JC, Abdala AP, Borgmann A, Rybak IA, Paton JF (2013) Brainstem respiratory networks: building blocks and microcircuits. Trends Neurosci 36:152-162.

Stanić D, Dhingra RR, Dutschmann M (2018) Expression of the transcription factor FOXP2 in brainstem respiratory circuits of adult rat is restricted to upper-airway pre-motor areas. Respir Physiol Neurobiol 250:14-18.

Stocchi F, Barbato L, Nordera G, Berardelli A, Ruggieri S (1998) Sleep disorders in Parkinson's disease. J Neurol 245:S15-S18.

Stornetta RL, Moreira TS, Takakura AC, Kang BJ, Chang DA, West GH, Brunet JF, Mulkey DK, Bayliss DA, Guyenet PG (2006) Expression of Phox $2 b$ by brainstem neurons involved in chemosensory integration in the adult rat. J Neurosci 26:10305-10314.

Takakura AC, Moreira TS, Colombari E, West GH, Stornetta RL, Guyenet PG (2006) Peripheral chemoreceptor inputs to retrotrapezoid nucleus (RTN) $\mathrm{CO}_{2}$-sensitive neurons in rats. J Physiol 572:503-523.

Tomita S, Oeda T, Umemura A, Kohsaka M, Park K, Yamamoto K, Sugiyama H, Sawada H (2018) Video-fluoroscopic swallowing study scale for predicting aspiration pneumonia in Parkinson's disease. PLoS One 13:e0197608.

Toor RUAS, Sun QJ, Kumar NN, Le S, Hildreth CM, Phillips JK, McMullan $S$ (2019) Neurons in the intermediate reticular nucleus coordinate postinspiratory activity, swallowing, and respiratory-sympathetic coupling in the rat. J Neurosci 39:9757-9766.

Tuppy M, Barna BF, Alves-Dos-Santos L, Britto LR, Chiavegatto S, Moreira TS, Takakura AC (2015) Respiratory deficits in a rat model of Parkinson's disease. Neuroscience 297:194-204.

Vann NC, Pham FD, Hayes JA, Kottick A, Del Negro CA (2016) Transient suppression of Dbx1 preBötzinger interneurons disrupts breathing in adult mice. PLoS One 11:e0162418.

Vann NC, Pham FD, Dorst KE, Del Negro CA (2018) Dbx1 pre-Bötzinger complex interneurons comprise the core inspiratory oscillator for breathing in unanesthetized adult mice. eNeuro 5:ENEURO.0130-18.2018.

Wang X, Hayes JA, Revill AL, Song H, Kottick A, Vann NC, LaMar MD, Picardo MC, Akins VT, Funk GD, Del Negro CA (2014) Laser ablation of Dbx1 neurons in the pre-Bötzinger complex stops inspiratory rhythm and impairs output in neonatal mice. Elife 3:e03427.

Withers PC (1977) Measurement of VO2, VCO2, and evaporative water loss with a flow-through mask. J Appl Physiol Respir Environ Exerc Physiol 42:120-123.

Zhang K, Chammas C, Soghomonian JJ (2015) Loss of glutamic acid decarboxylase (Gad67) in striatal neurons expressing the Drdrla dopamine receptor prevents L-DOPA-induced dyskinesia in 6-hydroxydopaminelesioned mice. Neuroscience 303:586-594.

Zhang LY, Liu WY, Kang WY, Yang Q, Wang XY, Ding JQ, Chen SD, Liu J (2016) Association of rapid eye movement sleep behavior disorder with sleep-disordered breathing in Parkinson's disease. Sleep Med 20:110-115. 